TRANSACTIONS OF THE

AMERICAN MATHEMATICAL SOCIETY

Volume 359, Number 11, November 2007, Pages 5383-5418

S 0002-9947(07)04217-1

Article electronically published on June 4, 2007

\title{
MULTIPLE ORTHOGONAL POLYNOMIALS AND A COUNTEREXAMPLE TO THE GAUDIN BETHE ANSATZ CONJECTURE
}

\author{
E. MUKHIN AND A. VARCHENKO
}

\begin{abstract}
Jacobi polynomials are polynomials whose zeros form the unique solution of the Bethe Ansatz equation associated with two $s l_{2}$ irreducible modules. We study sequences of $r$ polynomials whose zeros form the unique solution of the Bethe Ansatz equation associated with two highest weight $s l_{r+1}$ irreducible modules, with the restriction that the highest weight of one of the modules is a multiple of the first fundamental weight.

We describe the recursion which can be used to compute these polynomials. Moreover, we show that the first polynomial in the sequence coincides with the Jacobi-Piñeiro multiple orthogonal polynomial and others are given by Wronskian-type determinants of Jacobi-Piñeiro polynomials.

As a byproduct we describe a counterexample to the Bethe Ansatz Conjecture for the Gaudin model.
\end{abstract}

\section{INTRODUCTION}

1.1. Content of the paper. It is well known that zeros of the classical Jacobi orthogonal polynomial with parameters $\alpha, \beta$ satisfy a system of algebraic equations, which is known as the Bethe Ansatz equation of the Gaudin model associated to $s l_{2}$ and two irreducible modules with highest weights $-\alpha-1,-\beta-1$; see $[\mathrm{Sz}$, V2].

In this paper we study the Bethe Ansatz equation of the Gaudin model associated to $s l_{r+1}$ and two irreducible modules, one of which has an arbitrary highest weight and the highest weight of another one is a multiple of the first fundamental weight; see (6.2). In this case the dimensions of the spaces of singular vectors of a given weight are at most one-dimensional, and therefore we may expect an appearance of some orthogonal polynomials. In this paper we show that this is indeed so.

We write the weights as $\boldsymbol{m}=\left(m_{1}, \ldots, m_{r}\right)$ and $(k, 0, \ldots, 0)$; see (2.1). We show that then for each partition $\boldsymbol{l}=\left(l_{1}, l_{2} \ldots, l_{r}\right)$, where $l_{i}$ are non-negative integers, $l_{i} \geq l_{j}$, if $i>j$, and for generic values of the parameters $\boldsymbol{m}, k$, there exists a unique solution of the Bethe Ansatz equation. We consider polynomials $y_{1}, \ldots, y_{r}$, $\operatorname{deg} y_{i}=l_{i}$, whose zeros form such a solution.

We call parameters $\boldsymbol{m}, k, \boldsymbol{l}$ consistent if $m_{1}, \ldots, m_{r}, k$ are non-negative integers and (2.2) is satisfied. According to MV1, for consistent values of the parameters, the polynomials $y_{1}, \ldots, y_{r}$ can be computed from a certain space of polynomials

Received by the editors May 17, 2005 and, in revised form, September 15, 2005.

2000 Mathematics Subject Classification. Primary 82B23, 33C45.

The research of the first author was supported in part by NSF grant DMS-0140460.

The research of the second author was supported in part by NSF grant DMS-0244579.

(C)2007 American Mathematical Society Reverts to public domain 28 years from publication 
$V(\boldsymbol{m}, \boldsymbol{l}, k)$ of dimension $r+1$ which has only two singular points. Degrees of polynomials in $V(\boldsymbol{m}, \boldsymbol{l}, k)$ and the exponents at the singular points are given explicitly in terms of the weights $\boldsymbol{m}, k$ and the partition $\boldsymbol{l}$; see Lemma 2.2

We construct the spaces $V(\boldsymbol{m}, \boldsymbol{l}, k)$ recursively using explicit linear differential operators, see Corollary [3.6. and discover that $V(\boldsymbol{m}, \boldsymbol{l}, k)$ has a basis written in terms of multiple orthogonal polynomials called the Jacobi-Piñeiro polynomials; see Lemma 4.4 and Proposition 4.6.

The Jacobi-Piñeiro polynomial $\left[\mathrm{P}\right.$ is the unique monic polynomial of degree $l_{1}$ whose coefficients are rational functions of $\boldsymbol{m}, k$ and which is orthogonal to functions

$$
\underbrace{1, x, \ldots, x^{l_{1}-l_{2}-1}}_{l_{1}-l_{2}}, \underbrace{x^{-m_{2}-1}, x^{-m_{2}}, \ldots, x^{-m_{2}+l_{2}-l_{3}-2}}_{l_{r}}, \ldots,
$$

with respect to the scalar product given by

$$
(f(x), g(x))=\int_{0}^{1} f(x) g(x)(x-1)^{-k-1} x^{-m_{1}-1} d x
$$

if $m_{i}, k$ are all negative real numbers.

It follows that the polynomials $y_{1}, \ldots, y_{r}$ are Wronskians of the Jacobi-Piñeiro polynomials up to an explicit factor. In particular $y_{1}$ is exactly the Jacobi-Piñeiro polynomial. Using properties of multiple orthogonal polynomials we are able to prove that for general values of parameters $\boldsymbol{m}, k$ the corresponding Bethe vector is non-zero; see Theorem 6.8 .

Multiple orthogonal polynomials can be computed rather explicitly, and therefore we have explicit formulas for $y_{1}$. A similar computation allows us to obtain an explicit formula for $y_{r}$. Using these formulas, we find that for special values of the parameters, the $s l_{3}$ Bethe Ansatz equation has no solutions, and therefore we obtain a counterexample to the standard form of the Bethe Ansatz Conjecture; see Proposition 6.10.

We also describe the spaces of polynomials $U(\boldsymbol{m}, \boldsymbol{l}, k)$ which are dual to the spaces $V(\boldsymbol{m}, \boldsymbol{l}, k)$. These spaces $U(\boldsymbol{m}, \boldsymbol{l}, k)$ were considered in $\underline{\mathrm{Sc}}$ (with $x$ replaced by $-x)$. In $[\mathrm{Sc}$ the problem of finding an explicit basis was reduced to a linear system of equations of the size $\left(\sum_{i=1}^{r} m_{i}\right)-l_{1}$.

Thus, summarizing, we establish a relation between the Bethe Ansatz method and the theory of multiple orthogonal polynomials. We also establish recurrence relations between the vector spaces of polynomials of one variable, which have given dimension $r+1$, and exactly two singular points at $x=0$ and $x=1$. We start with a space of polynomials consisting of monomials and construct all other spaces using suitable first order linear polynomial differential operators. Moreover, we show that the differential operators form an extension of the $s l_{r+1}$ affine Weyl group. Those differential operators provide the Rodrigues-type explicit formulas for the bases of those spaces of polynomials.

A study similar to the one conducted in this paper can be done in the trigonometric case, related to multiple Laguerre polynomials, and also in the cases associated with the $X X X$ model and the $X X Z$ model, related to multiple little $q$-Jacobi polynomials studied in $[\mathrm{PV}]$. See in [MV3] related discussions of the $X X X$ model. 
Our paper is constructed as follows. In the rest of the introduction we state our main results in more detail for the cases of $s l_{2}$ and $s l_{3}$ and present our counterexample to the standard form of the Bethe Ansatz Conjecture. In Section 2 we define the spaces of polynomials $V(\boldsymbol{m}, \boldsymbol{l}, k)$ and $U(\boldsymbol{m}, \boldsymbol{l}, k)$. In Section 3 we construct those spaces recursively. In Section 4 we construct the basis of $V(\boldsymbol{m}, \boldsymbol{l}, k)$ in terms of Jacobi-Piñeiro polynomials. In Section 5 we give an explicit basis of $U(\boldsymbol{m}, \boldsymbol{l}, k)$. In Section 6 we use our results to study the Bethe Ansatz Conjecture. In Section 7 we present remarks on some integral identities and on an affine Weyl group action in our situation.

We thank Ken McLaughlin for useful discussions. The first author thanks the Max-Planck-Institute für Mathematik where part of this work was done for hospitality and a stimulating enviroment.

1.2. Jacobi polynomials with negative integer parameters. Let $m, l, k$ be non-negative integers such that

$$
k \geq l \geq 0, \quad m \geq l \geq 0 .
$$

Let

$$
\mathcal{D}(l, k)=x(x-1) \frac{d^{2}}{d x^{2}}-(k x+m(x-1)) \frac{d}{d x}+l(k+m+1-l) .
$$

Denote by $V(l, k)$ the two-dimensional complex vector space of solutions of the differential equation $\mathcal{D}(l, k) f=0$. The space $V(l, k)$ has the properties:

(i) The space $V(l, k)$ consists of polynomials of degrees $l$ and $k+m+1-l$, $l<k+m+1-l$.

(ii) There exists a polynomial in $V(l, k)$ which has a root at 0 of multiplicity $m+1$.

(iii) There exists a polynomial in $V(l, k)$ which has a root at 1 of multiplicity $k+1$.

These three properties define the two-dimensional space $V(l, k)$ uniquely.

Example. Polynomials 1 and $x^{m+1}$ form a basis in $V(0,0)$.

Let $y(l, k ; x)$ be the monic polynomial in $V(l, k)$ of degree $l$. Then

$$
y(l, k ; x)=P_{l}^{(\alpha, \beta)}(x),
$$

where $\alpha=-k-1, \beta=-m-1$, and $P_{l}^{(\alpha, \beta)}(x)$ is the monic Jacobi polynomial on the interval $[0,1]$.

A basis in $V(l, k)$ is formed by $P_{l}^{(-k-1,-m-1)}(x)$ and $x^{m+1} P_{k-l}^{(-k-1, m+1)}(x)$.

According to the Rodrigues formula the polynomial $y(l, k ; x)$ is equal to

$$
x^{m+1}(x-1)^{k+1} \frac{d^{l}}{d x^{l}}\left[x^{l-m-1}(x-1)^{l-k-1}\right]
$$

up to multiplication by a non-zero constant. 
Consider the first order linear differential operators

$$
\begin{aligned}
& D_{0}(l, k)=x(x-1) \frac{d}{d x}-l(x-1)-k-1, \\
& D_{1}(l, k)=x(x-1) \frac{d}{d x}-(k+m+1-l)(x-1)-k-1, \\
& D_{0}^{\vee}(l, k)=x \frac{d}{d x}-(k+m+1-l), \\
& D_{1}^{\vee}(l, k)=x \frac{d}{d x}-l .
\end{aligned}
$$

The operators commute:

$$
\begin{aligned}
D_{1}(l, k+1) D_{0}(l, k) & =D_{0}(l+1, k+1) D_{1}(l, k), \\
D_{1}^{\vee}(l, k-1) D_{0}^{\vee}(l, k) & =D_{0}^{\vee}(l-1, k-1) D_{1}^{\vee}(l, k) .
\end{aligned}
$$

The operators define isomorphisms:

$$
\begin{aligned}
& D_{0}(l, k): V(l, k) \rightarrow V(l, k+1), \\
& D_{1}(l, k): V(l, k) \rightarrow V(l+1, k+1), \\
& D_{0}^{\vee}(l, k): V(l, k) \rightarrow V(l, k-1), \\
& D_{1}^{\vee}(l, k): V(l, k) \rightarrow V(l-1, k-1)
\end{aligned}
$$

if the preimage and image spaces are defined, that is, if the corresponding parameters satisfy (1.1). In particular, the composition

$$
D_{1}(l-1, k-1) \cdots D_{1}(0, k-l) D_{0}(0, k-l-1) \cdots D_{0}(0,0)
$$

defines an isomorphism $V(0,0) \rightarrow V(l, k)$.

We have

$$
\begin{aligned}
y(l, k+1 ; x) & =\frac{k+m+2-2 l}{(k+m+2-l)(k+1-l)} D_{0}(l, k) y(l, k ; x), \\
y(l+1, k+1 ; x) & =-\frac{1}{k+m+1-2 l} D_{1}(l, k) y(l, k ; x) .
\end{aligned}
$$

For any $i$, denote by $W\left(f_{1}, \ldots, f_{i}\right)$ the Wronskian of functions $f_{1}, \ldots, f_{i}$ of $x$.

The formula

$$
\langle f, g\rangle_{l, k}=\frac{W(f, g)}{x^{k}(x-1)^{m}}, \quad f, g \in V(l, k),
$$

defines on $V(l, k)$ a non-degenerate skew-symmetric bilinear form.

The operators $D_{0}(l, k)$ and $D_{1}(l, k)$ are adjoint to the operators $-D_{0}^{\vee}(l, k+1)$ and $-D_{1}^{\vee}(l+1, k+1)$ respectively in the following sense:

$$
\begin{aligned}
\left\langle D_{0}(l, k) f, g\right\rangle_{l, k+1} & =-\left\langle f, D_{0}^{\vee}(l, k+1) g\right\rangle_{l, k}, \quad f \in V(l, k), g \in V(l, k+1), \\
\left\langle D_{1}(l, k) f, g\right\rangle_{l+1, k+1} & =-\left\langle f, D_{1}^{\vee}(l+1, k+1) g\right\rangle_{l, k}, f \in V(l, k), g \in V(l+1, k+1) .
\end{aligned}
$$

Let $t_{1}, \ldots, t_{l}$ be the roots of the polynomial $y(l, k ; x)$. The roots are pairwise distinct, different from 0 and 1 , and form a solution to the system of equations

$$
\frac{m}{t_{i}}+\frac{k}{t_{i}-1}-\sum_{j, j \neq i} \frac{2}{t_{i}-t_{j}}=0, \quad i=1, \ldots, l .
$$

If parameters $m, l, k$ satisfy (1.1), then system (1.3) has a unique solution $t_{1}, \ldots, t_{l}$ up to permutations of these numbers.

Formulas (1.2) give a recurrent way to generate solutions to (1.3). 
The coefficients of the polynomial $y(l, k ; x)$ are rational functions of $k, m$.

Assume that $\operatorname{Re} k<0$ and $\operatorname{Re} m<-1$. For polynomials $f, g$, set

$$
(f, g)_{k}=\int_{0}^{1} \frac{f(x) g(x)}{x^{m+2}(1-x)^{k+1}} d x .
$$

The operators $D_{0}(l, k)$ and $D_{1}(l, k)$ are adjoint to the operators $-D_{0}^{\vee}(l, k+1)$ and $-D_{1}^{\vee}(l+1, k+1)$ respectively in the following sense:

$$
\begin{aligned}
& \left(D_{0}(l, k) f, g\right)_{k+1}=-\left(f, D_{0}^{\vee}(l, k+1) g\right)_{k}, \\
& \left(D_{1}(l, k) f, g\right)_{k+1}=-\left(f, D_{1}^{\vee}(l+1, k+1) g\right)_{k} .
\end{aligned}
$$

We have

$$
\left(y(l, k ; x), x^{n}\right)_{k}=0, \quad 1 \leq n \leq l,
$$

and

$$
(y(l, k ; x), y(l, k ; x))_{k}=l ! \prod_{i=1}^{l} \frac{m+1-i}{(k+m+2-l-i)^{2}}(1,1)_{k-l},
$$

where

$$
(1,1)_{k-l}=\frac{\Gamma(-m-1) \Gamma(-k+l)}{\Gamma(-k-m-1+l)} .
$$

1.3. Three-dimensional spaces of polynomials with two singular points. The parameters $\boldsymbol{m}=\left(m_{1}, m_{2}\right), \boldsymbol{l}=\left(l_{1}, l_{2}\right) \in \mathbb{Z}_{\geq 0}^{2}, k \in \mathbb{Z}_{\geq 0}$ are called consistent if

$$
k \geq l_{1} \geq l_{2} \geq 0, \quad m_{1} \geq l_{1}-l_{2}, \quad m_{2} \geq l_{2} \text {. }
$$

Let $\boldsymbol{m}=\left(m_{1}, m_{2}\right), \boldsymbol{l}=\left(l_{1}, l_{2}\right), k$ be consistent. Let

$$
\mathcal{D}(\boldsymbol{l}, k)=x(x-1) \frac{d^{3}}{d x^{3}}-\left(\left(2 m_{1}+m_{2}\right)(x-1)+2 k x\right) \frac{d^{2}}{d x^{2}}+v_{1} \frac{d}{d x}+v_{0},
$$

where

$$
\begin{gathered}
v_{1}=-l_{2}^{2}+l_{2}\left(m_{2}+1\right)+l_{1}\left(k+m_{1}+1-l_{1}+l_{2}\right) \\
+\left(m_{1}+k\right)\left(m_{1}+m_{2}+k+1\right)-\frac{m_{1}\left(m_{1}+m_{2}+1\right)}{x}+\frac{k(k+1)}{x-1}, \\
v_{0}=-\frac{(k+1)\left(-l_{2}^{2}+l_{2}\left(m_{2}+1\right)+l_{1}\left(m_{1}+k+1-l_{1}+l_{2}\right)\right)}{x(x-1)} \\
-\frac{l_{1}\left(l_{2}-l_{1}+m_{1}+k+1\right)\left(2-l_{2}+m_{1}+k+m_{2}\right)}{x} .
\end{gathered}
$$

Denote by $V(\boldsymbol{l}, k)$ the three-dimensional complex vector space of solutions of the differential equation $\mathcal{D}(\boldsymbol{l}, k) f=0$. The space $V(\boldsymbol{l}, k)$ has the properties:

(i) The space $V(\boldsymbol{l}, k)$ consists of polynomials of degrees $l_{1}, m_{1}+1+k+l_{2}-l_{1}$, and $m_{1}+m_{2}+2+k-l_{2}$, where

$$
l_{1}<m_{1}+1+k+l_{2}-l_{1}<m_{1}+m_{2}+2+k-l_{2} .
$$

(ii) There exist three polynomials in $V(\boldsymbol{l}, k)$ which have a root at 0 of multiplicities $0, m_{1}+1, m_{1}+m_{2}+2$, respectively.

(iii) There exist three polynomials in $V(\boldsymbol{l}, k)$ which have a root at 1 of multiplicities $0, k+1, k+2$, respectively.

These three properties define the three-dimensional space $V(\boldsymbol{l}, k)$ uniquely. 
Example. Polynomials $1, x^{m_{1}+1}$, and $x^{m_{1}+m_{2}+2}$ form a basis in $V(\mathbf{0}, 0)$, where $\mathbf{0}=(0,0)$.

Set

$e_{0}(\boldsymbol{l}, k)=l_{1}, \quad e_{1}(\boldsymbol{l}, k)=m_{1}+1+k+l_{2}-l_{1}, \quad e_{2}(\boldsymbol{l}, k)=m_{1}+m_{2}+2+k-l_{2}$.

Set

$$
T_{1}(x)=x^{m_{1}}(x-1)^{k}, \quad T_{2}(x)=x^{m_{2}} .
$$

Let $f_{0}, f_{1}$ be the monic polynomials in $V(\boldsymbol{l}, k)$ of degrees $e_{0}(\boldsymbol{l}, k), e_{1}(\boldsymbol{l}, k)$, respectively. Set

$$
y_{1}(\boldsymbol{l}, k ; x)=f_{0}(x), \quad y_{2}(\boldsymbol{l}, k ; x)=\frac{W\left(f_{0}, f_{1}\right)}{\left(e_{0}(\boldsymbol{l}, k)-e_{1}(\boldsymbol{l}, k)\right) T_{1}(x)} .
$$

These are monic polynomials of degrees $l_{1}$ and $l_{2}$, respectively.

Let

$\mathcal{D}^{\vee}(\boldsymbol{l}, k)=x(x-1) \frac{d^{3}}{d x^{3}}-\left(\left(2 m_{2}+m_{1}\right)(x-1)+k x\right) \frac{d^{2}}{d x^{2}}+u_{1} \frac{d}{d x}+u_{0}$,

where

$$
\begin{aligned}
u_{1}= & l_{2}\left(l_{1}-l_{2}+m_{2}+1\right)+ \\
& \left(l_{2}+m_{2}+1\right)\left(m_{2}+m_{1}+k+2-l_{2}\right) \\
& -2-2 m_{2}-m_{1}-k-\frac{m_{2}\left(m_{1}+m_{2}+1\right)}{x}, \\
u_{0}= & \frac{l_{2}\left(l_{1}-l_{2}+m_{2}+1\right)\left(-2-m_{2}-m_{1}-k+l_{1}\right)}{x} .
\end{aligned}
$$

Denote by $U(\boldsymbol{l}, k)$ the three-dimensional complex vector space of solutions of the differential equation $\mathcal{D}^{\vee}(\boldsymbol{l}, k) f=0$. The space $U(\boldsymbol{l}, k)$ has the properties:

(i) The space $U(\boldsymbol{l}, k)$ consists of polynomials of degrees $l_{2}, m_{2}+1+l_{1}-l_{2}$, and $m_{1}+m_{2}+2+k-l_{1}$, where

$$
l_{2}<m_{2}+1+l_{1}-l_{2}<m_{1}+m_{2}+2+k-l_{1} .
$$

(ii) There exist three polynomials in $U(\boldsymbol{l}, k)$ which have a root at 0 of multiplicities $0, m_{2}+1, m_{1}+m_{2}+2$, respectively.

(iii) There exist three polynomials in $U(\boldsymbol{l}, k)$ which have a root at 1 of multiplicities $0,1, k+2$, respectively.

These three properties define the three-dimensional space $U(\boldsymbol{l}, k)$ uniquely.

Example. Polynomials $1, x^{m_{2}+1}$, and $x^{m_{1}+m_{2}+2}$ form a basis in $U(\mathbf{0}, 0)$.

Set

$$
e_{0}^{\vee}(\boldsymbol{l}, k)=l_{2}, \quad e_{1}^{\vee}(\boldsymbol{l}, k)=m_{2}+1+l_{1}-l_{2}, \quad e_{2}^{\vee}(\boldsymbol{l}, k)=m_{1}+m_{2}+2+k-l_{1} .
$$

For $f_{1}, f_{2}, f_{3} \in V(\boldsymbol{l}, k)$, set

$$
W_{V}^{\dagger}\left(f_{1}, f_{2}\right)=\frac{W\left(f_{1}, f_{2}\right)}{T_{1}}, \quad W_{V}^{\dagger}\left(f_{1}, f_{2}, f_{3}\right)=\frac{W\left(f_{1}, f_{2}\right)}{T_{1}^{2} T_{2}} .
$$

For given $f_{1}, f_{2}, f_{3}$, the function $W_{V}^{\dagger}\left(f_{1}, f_{2}\right)$ is a polynomial, and the function $W_{V}^{\dagger}\left(f_{1}, f_{2}, f_{3}\right)$ is constant. The map $\left(f_{1}, f_{2}, f_{3}\right) \mapsto W_{V}^{\dagger}\left(f_{1}, f_{2}, f_{3}\right)$ defines a volume form on $V(\boldsymbol{l}, k)$.

For $g_{1}, g_{2}, g_{3} \in U(\boldsymbol{l}, k)$, set

$$
W_{U}^{\dagger}\left(g_{1}, g_{2}\right)=\frac{W\left(g_{1}, g_{2}\right)}{T_{2}}, \quad W_{U}^{\dagger}\left(g_{1}, g_{2}, g_{3}\right)=\frac{W\left(g_{1}, g_{2}\right)}{T_{2}^{2} T_{1}} .
$$


For given $g_{1}, g_{2}, g_{3}$, the function $W_{U}^{\dagger}\left(g_{1}, g_{2}\right)$ is a polynomial, and the function $W_{U}^{\dagger}\left(g_{1}, g_{2}, g_{3}\right)$ is constant. The map $\left(g_{1}, g_{2}, g_{3}\right) \mapsto W_{U}^{\dagger}\left(g_{1}, g_{2}, g_{3}\right)$ defines a volume form on $U(\boldsymbol{l}, k)$.

We have

$$
\begin{aligned}
U(\boldsymbol{l}, k) & =\left\{W_{V}^{\dagger}\left(f_{1}, f_{2}\right) \mid f_{1}, f_{2} \in V(\boldsymbol{l}, k)\right\} \\
V(\boldsymbol{l}, k) & =\left\{W_{U}^{\dagger}\left(g_{1}, g_{2}\right) \mid g_{1}, g_{2} \in U(\boldsymbol{l}, k)\right\} .
\end{aligned}
$$

In particular, the polynomial $y_{2}(\boldsymbol{l}, k ; x)$ defined in (1.5) is the unique monic polynomial in $U(\boldsymbol{l}, k)$ of degree $l_{2}$.

The spaces $V(\boldsymbol{l}, k)$ and $U(\boldsymbol{l}, k)$ are dual with respect to the following pairing:

$$
\langle f, g\rangle_{l, k}=W_{V}^{\dagger}\left(f, f_{1}, f_{2}\right) \quad \text { if } g=W_{V}^{\dagger}\left(f_{1}, f_{2}\right) .
$$

In particular, we have

$$
\left\langle y_{1}(\boldsymbol{l}, k ; x), y_{2}(\boldsymbol{l}, k ; x)\right\rangle_{\boldsymbol{l}, k}=0 .
$$

Consider the first order linear differential operators

$$
\begin{aligned}
D_{i}(\boldsymbol{l}, k) & =x(x-1) \frac{d}{d x}-e_{i}(\boldsymbol{l}, k)(x-1)-k-1, \\
D_{i}^{\vee}(\boldsymbol{l}, k) & =x \frac{d}{d x}-e_{2-i}^{\vee}(\boldsymbol{l}, k),
\end{aligned}
$$

$i=0,1,2$. These obey the linear relations:

$$
\begin{gathered}
A_{12}(\boldsymbol{l}, k) D_{0}(\boldsymbol{l}, k)+A_{20}(\boldsymbol{l}, k) D_{1}(\boldsymbol{l}, k)+A_{01}(\boldsymbol{l}, k) D_{2}(\boldsymbol{l}, k)=0, \\
A_{12}(\boldsymbol{l}, k) D_{0}^{\vee}(\boldsymbol{l}, k)+A_{20}(\boldsymbol{l}, k) D_{1}^{\vee}(\boldsymbol{l}, k)+A_{01}(\boldsymbol{l}, k) D_{2}^{\vee}(\boldsymbol{l}, k)=0,
\end{gathered}
$$

where $A_{i j}(\boldsymbol{l}, k)=e_{i}(\boldsymbol{l}, k)-e_{j}(\boldsymbol{l}, k)$. Set

$$
\mathbf{1}_{0}=(0,0), \quad \mathbf{1}_{1}=(1,0), \quad \mathbf{1}_{2}=(1,1) .
$$

The operators commute:

$$
\begin{aligned}
D_{i}\left(\boldsymbol{l}+\mathbf{1}_{j}, k+1\right) D_{j}(\boldsymbol{l}, k) & =D_{j}\left(\boldsymbol{l}+\mathbf{1}_{i}, k+1\right) D_{i}(\boldsymbol{l}, k) \\
D_{i}^{\vee}\left(\boldsymbol{l}-\mathbf{1}_{j}, k-1\right) D_{j}^{\vee}(\boldsymbol{l}, k) & =D_{j}^{\vee}\left(\boldsymbol{l}-\mathbf{1}_{i}, k-1\right) D_{i}^{\vee}(\boldsymbol{l}, k)
\end{aligned}
$$

for all $i, j$.

The operators define isomorphisms:

$$
\begin{aligned}
D_{i}(\boldsymbol{l}, k): & : V(\boldsymbol{l}, k) \rightarrow V\left(\boldsymbol{l}+\mathbf{1}_{i}, k+1\right), \\
D_{i}^{\vee}(\boldsymbol{l}, k): & : U(\boldsymbol{l}, k) \rightarrow U\left(\boldsymbol{l}-\mathbf{1}_{i}, k-1\right),
\end{aligned}
$$

for $i=0,1,2$, if the preimage and image spaces are defined, that is, if the corresponding parameters satisfy (1.4). In particular, the composition

$$
\begin{array}{r}
D_{2}\left(\left(l_{1}-1, l_{2}-1\right), k-1\right) \cdots D_{2}\left(\left(l_{1}-l_{2}, 0\right), k-l_{2}\right) D_{1}\left(\left(l_{1}-l_{2}-1,0\right), k-l_{2}-1\right) \\
\cdots D_{1}\left((0,0), k-l_{1}\right) D_{0}\left((0,0), k-l_{1}-1\right) \cdots D_{0}((0,0), 0)
\end{array}
$$

defines an isomorphism $V(\mathbf{0}, 0) \rightarrow V(\boldsymbol{l}, k)$, and the composition

$$
\begin{aligned}
& D_{0}^{\vee}((0,0), 1) \cdots D_{0}^{\vee}\left((0,0), k-l_{1}\right) D_{1}^{\vee}\left((1,0), k-l_{1}+1\right) \\
& \quad \cdots D_{1}^{\vee}\left(\left(l_{1}-l_{2}, 0\right), k-l_{2}\right) D_{2}^{\vee}\left(\left(l_{1}-l_{2}+1,1\right), k-l_{2}+1\right) \cdots D_{2}^{\vee}\left(\left(l_{1}, l_{2}\right), k\right)
\end{aligned}
$$

defines an isomorphism $U(\boldsymbol{l}, k) \rightarrow U(\mathbf{0}, 0)$. 
Set $l_{0}=k, l_{3}=0$, and

$$
\begin{gathered}
A_{0}(\boldsymbol{l}, k)=\prod_{i=0}^{2} \frac{k+\sum_{j=1}^{i} m_{j}-l_{1}+i+1}{k+\sum_{j=1}^{i} m_{j}-l_{1}+i+1-l_{i}+l_{i+1}}, \\
A_{1}(\boldsymbol{l}, k)=2 l_{1}-l_{2}-k-m_{1}-1, \quad A_{2}(\boldsymbol{l}, k)=l_{1}+l_{2}-k-m_{1}-m_{2}-2, \\
A_{0}^{\vee}(\boldsymbol{l}, k)=l_{1}+l_{2}-k-m_{1}-m_{2}-2, A_{1}^{\vee}(\boldsymbol{l}, k)=2 l_{2}-l_{1}-m_{2}-1, \\
A_{2}^{\vee}(\boldsymbol{l}, k)=-\prod_{i=0}^{2} \frac{\sum_{j=3-i}^{2} m_{j}-l_{2}+i}{\sum_{j=3-i}^{2} m_{j}-l_{2}+i+1-l_{2-i}+l_{3-i}} .
\end{gathered}
$$

Then for $i=0,1,2$, we have

$$
\begin{array}{ll}
A_{i}(\boldsymbol{l}, k) y_{1}\left(\boldsymbol{l}+\mathbf{1}_{i}, k+1 ; x\right) & =D_{i}(\boldsymbol{l}, k) y_{1}(\boldsymbol{l}, k ; x), \\
A_{i}^{\vee}(\boldsymbol{l}, k) y_{2}\left(\boldsymbol{l}-\mathbf{1}_{i}, k-1 ; x\right) & =D_{i}^{\vee}(\boldsymbol{l}, k) y_{2}(\boldsymbol{l}, k ; x) .
\end{array}
$$

Here is a Rodrigues-type formula for $y_{1}(\boldsymbol{l}, k ; x)$ :

$$
\begin{array}{r}
x^{m_{1}+m_{2}+2}(x-1)^{k+1}\left(\frac{d}{d x}\right)^{l_{2}}\left[x^{-m_{2}+l_{2}-1}\left(\frac{d}{d x}\right)^{l_{1}-l_{2}}\left[x^{l_{1}-l_{2}-m_{1}-1}(x-1)^{l_{1}-k-1}\right]\right] \\
=c y_{1}(\boldsymbol{l}, k ; x),
\end{array}
$$

where $c$ is a non-zero constant. If

$$
y_{1}(\boldsymbol{l}, k ; x)=\sum_{n=0}^{l_{1}} a_{n}(\boldsymbol{l}, k)(x-1)^{n}, \quad y_{2}(\boldsymbol{l}, k ; x)=\sum_{n=0}^{l_{2}} b_{n}(\boldsymbol{l}, k) x^{n}
$$

with $a_{l_{1}}(\boldsymbol{l}, k)=1, a_{-1}(\boldsymbol{l}, k)=a_{l_{1}+1}(\boldsymbol{l}, k)=0$, and $b_{l_{2}}(\boldsymbol{l}, k)=1, b_{l_{2}+1}(\boldsymbol{l}, k)=0$, then

$$
\begin{aligned}
& a_{n}\left(\boldsymbol{l}+\mathbf{1}_{i}, k+1\right)=-\frac{k+1+n}{A_{i}(\boldsymbol{l}, k)} a_{n}(\boldsymbol{l}, k)+\frac{n-1-e_{i}(\boldsymbol{l}, k)}{A_{i}(\boldsymbol{l}, k)} a_{n-1}(\boldsymbol{l}, k), \\
& b_{n}\left(\boldsymbol{l}+\mathbf{1}_{i}, k+1\right)=\frac{A_{i}^{\vee}\left(\boldsymbol{l}+\mathbf{1}_{i}, k+1\right)}{n-e_{2-i}^{\vee}\left(\boldsymbol{l}+\mathbf{1}_{i}, k+1\right)} b_{n}(\boldsymbol{l}, k) .
\end{aligned}
$$

In particular,

$$
b_{n}(\boldsymbol{l}, k)=\left(\begin{array}{c}
l_{2} \\
n
\end{array}\right) \prod_{i=0}^{l_{2}-n-1} \prod_{j=1}^{2} \frac{\sum_{s=3-j}^{2} m_{s}-l_{2}+i+j}{\sum_{s=3-j}^{2} m_{s}-l_{2}+i+j+1+l_{2-j}-l_{3-j}},
$$

where $l_{0}=k$.

We have

$$
\begin{aligned}
& A_{12}(\boldsymbol{l}, k) A_{0}(\boldsymbol{l}, k) y_{1}\left(\boldsymbol{l}+\mathbf{1}_{0}, k+1 ; x\right) \\
& +A_{20}(\boldsymbol{l}, k) A_{1}(\boldsymbol{l}, k) y_{1}\left(\boldsymbol{l}+\mathbf{1}_{1}, k+1 ; x\right) \\
& +A_{01}(\boldsymbol{l}, k) A_{2}(\boldsymbol{l}, k) y_{1}\left(\boldsymbol{l}+\mathbf{1}_{2}, k+1 ; x\right)=0, \\
& A_{12}(\boldsymbol{l}, k) A_{0}^{\vee}(\boldsymbol{l}, k) y_{2}\left(\boldsymbol{l}-\mathbf{1}_{0}, k-1 ; x\right) \\
& +A_{20}(\boldsymbol{l}, k) A_{1}^{\vee}(\boldsymbol{l}, k) y_{2}\left(\boldsymbol{l}-\mathbf{1}_{1}, k-1 ; x\right) \\
& +A_{01}(\boldsymbol{l}, k) A_{2}^{\vee}(\boldsymbol{l}, k) y_{2}\left(\boldsymbol{l}-\mathbf{1}_{2}, k-1 ; x\right)=0,
\end{aligned}
$$

and

$$
y_{1}(\boldsymbol{l}, k ; 0) \neq 0, \quad y_{1}(\boldsymbol{l}, k ; 1) \neq 0, \quad y_{2}(\boldsymbol{l}, k ; 1) \neq 0 .
$$


Example. If $\boldsymbol{l}=(2,1)$, then

$$
\begin{aligned}
y_{1}((2,1), k ; x)=( & -1)^{2}+\frac{(k-1)\left(2 k+2 m_{1}+m_{2}\right)}{\left(m_{1}+k-1\right)\left(m_{1}+m_{2}+k\right)}(x-1) \\
& +\frac{k(k-1)}{\left(m_{2}+m_{1}+k\right)\left(m_{1}+k-1\right)}, \\
y_{2}((2,1), k ; x)=x & -\frac{m_{2}\left(m_{1}+m_{2}+1\right)}{\left(m_{2}+2\right)\left(k+m_{1}+m_{2}\right)} .
\end{aligned}
$$

In particular, if $\left(2 m_{1}+m_{2}\right)^{2}+k\left(4 m_{1}-m_{2}^{2}\right)=0$. Then

$$
y_{1}((2,1), k ; x)=y_{2}((2,1), k ; x)^{2} .
$$

For $i=0,1,2$, the operator $D_{i}(\boldsymbol{l}, k)$ is adjoint to the operator $-D_{i}^{\vee}\left(\boldsymbol{l}+\mathbf{1}_{i}, k+1\right)$ in the following sense:

$$
\left\langle D_{i}(\boldsymbol{l}, k) f, g\right\rangle_{\boldsymbol{l}+\mathbf{1}_{i}, k+1}=-\left\langle f, D_{i}^{\vee}\left(\boldsymbol{l}+\mathbf{1}_{i}, k+1\right) g\right\rangle_{\boldsymbol{l}, k}
$$

if $f \in V(\boldsymbol{l}, k)$, and $g \in U\left(\boldsymbol{l}+\mathbf{1}_{i}, k+1\right)$.

Let $t_{1}, \ldots, t_{l_{1}}$ be the roots of the polynomial $y_{1}(\boldsymbol{l}, k ; x)$. Let $s_{1}, \ldots, s_{l_{2}}$ be the roots of the polynomial $y_{2}(\boldsymbol{l}, k ; x)$. Assume that $y_{1}(\boldsymbol{l}, k ; x)$ and $y_{2}(\boldsymbol{l}, k ; x)$ do not have multiple roots. Then the roots satisfy the system of equations

$$
\begin{aligned}
\frac{m_{1}}{t_{i}}+\frac{k}{t_{i}-1}-\sum_{j, j \neq i} \frac{2}{t_{i}-t_{j}}+\sum_{j} \frac{1}{t_{i}-s_{j}}=0, \quad i=1, \ldots, l_{1}, \\
\frac{m_{2}}{s_{i}}-\sum_{j, j \neq i} \frac{2}{s_{i}-s_{j}}+\sum_{j} \frac{1}{s_{i}-t_{j}}=0, \quad i=1, \ldots, l_{2} .
\end{aligned}
$$

If parameters $\boldsymbol{m}, \boldsymbol{l}, k$ are consistent, then system (1.3) has at most one solution $t_{1}, \ldots, t_{l_{1}}, s_{1}, \ldots, s_{l_{2}}$ up to permutations of the first and second groups of these numbers.

If parameters $\boldsymbol{l}$ are fixed, then for almost all $\boldsymbol{m}, k$ such that $\boldsymbol{m}, \boldsymbol{l}, k$ are consistent, the polynomials $y_{1}(\boldsymbol{l}, k ; x)$ and $y_{2}(\boldsymbol{l}, k ; x)$ do not have multiple roots.

Formulas (1.6) give a recurrent way to generate solutions to (1.7).

The coefficients of the polynomials $y_{1}(\boldsymbol{l}, k ; x)$ and $y_{2}(\boldsymbol{l}, k ; x)$ are rational functions of $k, \boldsymbol{m}$.

Assume that $\operatorname{Re} k<0$ and $\operatorname{Re}\left(m_{1}+m_{2}\right)<-2$. For polynomials $f, g$, set

$$
(f, g)_{k}=\int_{0}^{1} \frac{f(x) g(x)}{x^{m_{1}+m_{2}+3}(1-x)^{k+1}} d x .
$$

For $i=0,1,2$, the operator $D_{i}(\boldsymbol{l}, k)$ is adjoint to the operator $-D_{i}^{\vee}\left(\boldsymbol{l}+\mathbf{1}_{i}, k+1\right)$ in the following sense:

$$
\left(D_{i}(\boldsymbol{l}, k) f, g\right)_{k+1}=-\left(f, D_{i}^{\vee}\left(\boldsymbol{l}+\mathbf{1}_{i}, k+1\right) g\right)_{k} .
$$

We have

$$
\begin{aligned}
\int_{0}^{1} \frac{y_{1}(\boldsymbol{l}, k ; x) x^{n}}{x^{m_{1}+m_{2}+3}(1-x)^{k+1}} d x & =0, & & n=1, \ldots, l_{2}, \\
\int_{0}^{1} \frac{y_{1}(\boldsymbol{l}, k ; x) x^{n}}{x^{m_{1}+2}(1-x)^{k+1}} d x & =0, & & n=1, \ldots, l_{1}-l_{2} .
\end{aligned}
$$


We also have

$$
\left(y_{1}(\boldsymbol{l}, k ; x), y_{2}(\boldsymbol{l}, k ; x)\right)_{k}=C(\boldsymbol{l}, k),
$$

where

$$
\begin{aligned}
& C(\boldsymbol{l}, k)=l_{2} ! \frac{\Gamma\left(-k+l_{1}\right) \Gamma\left(-m_{1}-m_{2}-2\right)}{\Gamma\left(-k-m_{1}-m_{2}-2+l_{1}\right)} \times \\
& \prod_{i=0}^{l_{2}-1} \frac{\left(m_{2}-i\right)\left(m_{1}+m_{2}+1-i\right)}{\left(m_{2}+l_{1}-l_{2}+1-i\right)\left(k+m_{1}+m_{2}+2-l_{1}-i\right)^{2}} \prod_{i=0}^{l_{1}-l_{2}-1} \frac{m_{2}+2+i}{k+m_{1}+1-l_{1}-i} .
\end{aligned}
$$

In the next formulas we include $\boldsymbol{m}$ in the notation for $y_{1}(\boldsymbol{l}, k ; x)$ and $V(\boldsymbol{l}, k)$. Then for consistent $\boldsymbol{m}, \boldsymbol{l}, k$, the space $V(\boldsymbol{m}, \boldsymbol{l}, k)$ has a basis formed by the polynomials

$$
\begin{aligned}
& y_{1}\left(\left(m_{1}, m_{2}\right),\left(l_{1}, l_{2}\right), k ; x\right), \\
& \quad x^{m_{1}+1} y_{1}\left(\left(-m_{1}-2, m_{1}+m_{2}+1\right),\left(k+l_{2}-l_{1}, l_{2}\right), k ; x\right), \\
& \quad x^{m_{1}+m_{2}+2} y_{1}\left(\left(m_{2},-m_{1}-m_{2}-3\right),\left(k+l_{2}-l_{1}, k-l_{1}\right), k ; x\right) .
\end{aligned}
$$

1.4. Application to the Bethe Ansatz in the Gaudin model. We let $\mathfrak{g}=$ $\mathfrak{n}_{+} \oplus \mathfrak{h} \oplus \mathfrak{n}_{-}$be a simple Lie algebra, $\quad \alpha_{1}, \ldots, \alpha_{r} \in \mathfrak{h}^{*}$ simple roots.

For a $\mathfrak{g}$-module $L$ and $\mu \in \mathfrak{h}^{*}$ denote by $L[\mu]$ the weight subspace of $L$ of weight $\mu$ and by $\operatorname{Sing} L[\mu]$ the subspace of singular vectors of weight $\mu$.

Let $n$ be a positive integer and $\boldsymbol{\Lambda}=\left(\Lambda_{1}, \ldots, \Lambda_{n}\right), \Lambda_{i} \in \mathfrak{h}^{*}$, a set of integral dominant weights. For a weight $\mu \in \mathfrak{h}^{*}$, let $L_{\mu}$ be the irreducible $\mathfrak{g}$-module with highest weight $\mu$. Denote by $L_{\boldsymbol{\Lambda}}$ the tensor product $L_{\Lambda_{1}} \otimes \cdots \otimes L_{\Lambda_{n}}$.

Let $\boldsymbol{z}=\left(z_{1}, \ldots, z_{n}\right)$ be a point in $\mathbb{C}^{n}$ with distinct coordinates. Introduce the linear operators $K_{1}(\boldsymbol{z}), \ldots, K_{n}(\boldsymbol{z})$ on $L_{\boldsymbol{\Lambda}}$ by the formula

$$
K_{i}(\boldsymbol{z})=\sum_{j, j \neq i} \frac{\Omega^{(i, j)}}{z_{i}-z_{j}}, \quad i=1, \ldots, n .
$$

The operators are called the Gaudin Hamiltonians of the Gaudin model associated with $L_{\boldsymbol{\Lambda}}$. The Hamiltonians commute.

The problem is to diagonalize simultaneously the Hamiltonians; see $\mathrm{B}, \mathrm{BF}, \mathrm{FFR}$, G, MV1, RV, ScV, , V2.

The Hamiltonians commute with the $\mathfrak{g}$-action on $L_{\boldsymbol{\Lambda}}$. It is enough to diagonalize the Hamiltonians on the subspaces of singular vectors, Sing $L_{\boldsymbol{\Lambda}}[\mu] \subset L_{\boldsymbol{\Lambda}}$.

The eigenvectors of the Gaudin Hamiltonians are constructed by the Bethe Ansatz method.

Fix $\mu=\sum_{s=1}^{n} \Lambda_{s}-\sum_{i=1}^{r} l_{i} \alpha_{i}$ where $l_{1}, \ldots, l_{r}$ are non-negative integers. Set

$$
\boldsymbol{t}=\left(t_{1}^{(1)}, \ldots, t_{l_{1}}^{(1)}, t_{1}^{(2)}, \ldots, t_{l_{2}}^{(2)}, \ldots, t_{1}^{(r)}, \ldots, t_{l_{r}}^{(r)}\right) .
$$

One defines a suitable rational function $w(\boldsymbol{t}, \boldsymbol{z})$ with values in $L_{\boldsymbol{\Lambda}}[\mu]$ as in [RV]; cf. [MV2, RSV]. The function is symmetric with respect to the group $\boldsymbol{\Sigma}_{\boldsymbol{l}}=$ $\Sigma_{l_{1}} \times \cdots \times \Sigma_{l_{r}}$ of permutations of coordinates $t_{j}^{(i)}$ with the same upper index. One considers the system of equations

$$
-\sum_{s=1}^{n} \frac{\left(\Lambda_{s}, \alpha_{i}\right)}{t_{j}^{(i)}-z_{s}}+\sum_{s, s \neq i} \sum_{k=1}^{l_{s}} \frac{\left(\alpha_{s}, \alpha_{i}\right)}{t_{j}^{(i)}-t_{k}^{(s)}}+\sum_{s, s \neq j} \frac{\left(\alpha_{i}, \alpha_{i}\right)}{t_{j}^{(i)}-t_{s}^{(i)}}=0,
$$


where $i=1, \ldots, r$ and $j=1, \ldots, l_{i}$. The system is symmetric with respect to $\Sigma_{l}$. One shows that if $\boldsymbol{t}^{0}$ is a solution to (1.8), then $w\left(\boldsymbol{t}^{0}, \boldsymbol{z}\right)$ belongs to $\operatorname{Sing} L_{\boldsymbol{\Lambda}}[\mu]$ and $w\left(\boldsymbol{t}^{0}, \boldsymbol{z}\right)$ is an eigenvector of the Hamiltonians $K_{1}(\boldsymbol{z}), \ldots, K_{n}(\boldsymbol{z})$; see [RV].

This method of finding eigenvectors is called the Bethe Ansatz method. System (1.8) is called the Bethe Ansatz equation, and the vector $w\left(\boldsymbol{t}^{0}, \boldsymbol{z}\right)$ is called a Bethe vector.

The Bethe Ansatz Conjecture says that if $\Lambda_{1}, \ldots, \Lambda_{n}$ are integral dominant, the weight $\mu$ is integral dominant, and $z_{1}, \ldots, z_{n}$ are generic, then the Bethe vectors form a basis in Sing $L_{\boldsymbol{\Lambda}}[\mu]$. In particular, the conjecture implies that the number of $\boldsymbol{\Sigma}_{\boldsymbol{l}}$-orbits of solutions to (1.8) is not less than the dimension of $\operatorname{Sing} L_{\boldsymbol{\Lambda}}[\mu]$.

Assume that $\mathfrak{g}=s l_{3}, n=2, z_{1}=0, z_{2}=1$. Assume that for some non-negative integers $k, m_{1}, m_{2}$, the weights $\Lambda_{1}$ and $\Lambda_{2}$ are such that

$$
\left(\Lambda_{1}, \alpha_{1}\right)=m_{1}, \quad\left(\Lambda_{1}, \alpha_{2}\right)=m_{2}, \quad\left(\Lambda_{2}, \alpha_{1}\right)=k, \quad\left(\Lambda_{2}, \alpha_{2}\right)=0 .
$$

Then system (1.8) becomes system (1.7).

Let $\mu=\Lambda_{1}+\Lambda_{2}-l_{1} \alpha_{1}-l_{2} \alpha_{2}$ be integral dominant. Then the space of highest weight vectors $\operatorname{Sing} L_{\Lambda_{1}} \otimes L_{\Lambda_{2}}[\mu]$ is one-dimensional, if parameters $\boldsymbol{m}, \boldsymbol{l}, k$ are consistent, and is zero-dimensional otherwise.

It was shown in MV1] that if $\mu$ is integral dominant and the numbers $t_{1}, \ldots, t_{l_{1}}$ and $s_{1}, \ldots, s_{l_{2}}$ form a solution to (1.7), then $\boldsymbol{m}, \boldsymbol{l}, k$ are consistent and

$$
\prod_{i=1}^{l_{1}}\left(x-t_{i}\right)=y_{1}(\boldsymbol{l}, k ; x), \quad \prod_{i=1}^{l_{2}}\left(x-s_{i}\right)=y_{2}(\boldsymbol{l}, k ; x) .
$$

Then we obtain, see the example in Section 1.3 ,

A counterexample to the Bethe Ansatz Conjecture. Let $\boldsymbol{l}=(2,1)$. Let $\boldsymbol{m}, \boldsymbol{l}, k$ be consistent and

$$
\left(2 m_{1}+m_{2}\right)^{2}+k\left(4 m_{1}-m_{2}^{2}\right)=0 .
$$

Then

$$
y_{1}((2,1), k ; x)=y_{2}((2,1), k ; x)^{2},
$$

and the roots of these two polynomials do not form a solution to (1.7). Hence system (1.7) does not have solutions in this case. Hence there is no Bethe vector to generate the one-dimensional space $\operatorname{Sing}\left(L_{\Lambda_{1}} \otimes L_{\Lambda_{2}}\right)[\mu]$.

For example, the parameters $\boldsymbol{m}=(2,3), k=49, \boldsymbol{l}=(2,1)$ are consistent and satisfy (1.9).

\section{Spaces of POLYNOMials}

In this section we define our main objects: the spaces of polynomials with two singular points 0,1 and special exponents at 1 .

2.1. An $s l_{r+1}$ lemma. Fix a natural number $r$ and choose non-negative integers $m_{1}, \ldots, m_{r}$. Choose a non-negative integer $k$.

Consider the complex Lie algebra $s l_{r+1}$. Let $\mathfrak{h}$ be its Cartan subalgebra, let $\alpha_{1}, \ldots, \alpha_{r} \in \mathfrak{h}^{*}$ be its simple roots, and $($,$) the scalar product on \mathfrak{h}^{*}$ such that $\left(\alpha_{i}, \alpha_{i}\right)=2$. For a weight $\Lambda \in \mathfrak{h}^{*}$ denote by $L_{\Lambda}$ the irreducible $s l_{r+1}$ module with highest weight $\Lambda$. 
Let the weights $\Lambda_{1}, \Lambda_{2}$ be such that the scalar products with simple roots are given by

$$
\left(\Lambda_{1}, \alpha_{s}\right)=m_{s}, \quad\left(\Lambda_{2}, \alpha_{s}\right)=\delta_{s 1} k
$$

where $s=1, \ldots, r$. Thus $L_{\Lambda_{2}}$ is the $k$-th symmetric power of the vector representation and $L_{\Lambda_{1}}$ can be any irreducible finite-dimensional $s l_{r+1}$ module.

We describe the decomposition of the tensor product $L_{\Lambda_{1}} \otimes L_{\Lambda_{2}}$.

Lemma 2.1. We have an isomorphism of $s l_{r+1}$ modules:

$$
L_{\Lambda_{1}} \otimes L_{\Lambda_{2}}=\bigoplus L_{\Lambda_{1}+\Lambda_{2}-\sum_{s=1}^{r} l_{s} \alpha_{s}},
$$

where the sum is over non-negative integers $l_{1}, \ldots, l_{r}$ such that

$$
k \geq l_{1} \geq l_{2} \geq \cdots \geq l_{r} \geq 0, \quad l_{s}-l_{s+1} \leq m_{s} \quad(s=1, \ldots, r) .
$$

In particular the multiplicity of every module in the right hand side is one.

We use the notation $\boldsymbol{l}=\left(l_{1}, \ldots, l_{r}\right)$ and $\boldsymbol{m}=\left(m_{1}, \ldots, m_{r}\right)$. We call $\boldsymbol{l}$ a partition if $l_{i}$ are integers and $l_{1} \geq l_{2} \geq \cdots \geq l_{r} \geq 0$. We set

$$
l_{0}=k, \quad l_{r+1}=0 .
$$

We call parameters $\boldsymbol{m}, \boldsymbol{l}, k$ consistent if all $m_{i}, l_{i}$ and $k$ are non-negative integers satisfying inequalities (2.2).

2.2. Definition of spaces of polynomials $V(\boldsymbol{m}, \boldsymbol{l}, k)$ and $U(\boldsymbol{m}, \boldsymbol{l}, k)$. Fix a positive number $r$. Let $V$ be an $(r+1)$-dimensional complex vector space of polynomials of one variable $x$. Assume that $V$ has no base points; i.e., for any $z \in \mathbb{C}$ there exists $f \in V$ such that $f(z) \neq 0$.

The Wronskian polynomial $W(V)$ of $V$ is the Wronskian of any basis $f_{1}, \ldots, f_{r+1}$ of $V$ :

$$
W(V)=W\left(f_{1}, \ldots, f_{r+1}\right)=\operatorname{det}\left(\frac{d^{i}}{d x^{i}} f_{j}\right) .
$$

The Wronskian polynomial $W(V)$ is defined up to multiplication by a non-zero complex number.

A point $z \in \mathbb{C}$ is called a singular point of $V$ if $z$ is a root of $W(V)$.

For any $z \in \mathbb{C}$ there exist unique non-negative integers $n_{1}(z), \ldots, n_{r}(z)$ such that for any $f \in V$ the order of zero of $f$ at $z$ belongs to the set

$$
\left\{0,1+n_{1}(z), 2+n_{1}(z)+n_{2}(z), \ldots, r+\sum_{s=1}^{r} n_{s}(z)\right\} .
$$

A point $z \in \mathbb{C}$ is a singular point of $V$ if and only if at least one of the numbers $n_{1}(z), \ldots, n_{r}(z)$ is positive. The set (2.3) is called the set of exponents of $V$ at $z$.

For $z \in \mathbb{C}$ we define a full flag in $V$ by

$$
\mathcal{F}(z)=\left\{0=F_{0}(z) \subset F_{1}(z) \subset F_{2}(z) \subset \cdots \subset F_{r+1}(z)=V\right\},
$$

where the $i$-dimensional subspace $F_{i}(z)$ consists of all polynomials with $\operatorname{root} x=z$ of order at least $r-i+1+\sum_{s=1}^{r-i+1} n_{s}(z)$. We call the flag $\mathcal{F}(z)$ the flag of $V$ at $z$. 
Similarly, there exist unique non-negative integers $n_{1}(\infty), \ldots, n_{r}(\infty), l$ such that for any $f \in V$ the degree of $f$ belongs to the set

$$
\left\{l, l+1+n_{1}(\infty), l+2+n_{1}(\infty)+n_{2}(\infty), \ldots, l+r+\sum_{s=1}^{r} n_{s}(\infty)\right\} .
$$

The set (2.4) is called the set of exponents of $V$ at infinity.

We define a full flag in $V$ by

$$
\mathcal{F}(\infty)=\left\{0=F_{0}(\infty) \subset F_{1}(\infty) \subset F_{2}(\infty) \subset \cdots \subset F_{r+1}(\infty)=V\right\}
$$

where the $i$-dimensional subspace $F_{i}(\infty)$ consists of all polynomials of degree no greater than $l+i-1+\sum_{s=1}^{i-1} n_{s}(\infty)$. We call the flag $\mathcal{F}(\infty)$ the flag of $V$ at infinity.

We have

$$
W(V)=\prod_{z \in \mathbb{C}}(x-z)^{\sum_{s=1}^{r}(r+1-s) n_{s}(z)},
$$

and therefore the following relation:

$$
\sum_{z \in \mathbb{C}} \sum_{s=1}^{r}(r+1-s) n_{s}(z)=(r+1) l+\sum_{s=1}^{r}(r+1-s) n_{s}(\infty) .
$$

Given a full flag $\mathcal{F}=\left\{0=F_{0} \subset F_{1} \subset \cdots \subset F_{r+1}=V\right\}$ we call a basis $\left\{f_{1}, \ldots, f_{r+1}\right\}$ of $V$ compatible with flag $\mathcal{F}$ if $\left\{f_{1}, \ldots, f_{s}\right\}$ is a basis of $F_{s}$ for $s=$ $1, \ldots, r$.

We use the notation $\boldsymbol{n}(\infty)=\left(n_{1}(\infty), \ldots, n_{r}(\infty)\right), \boldsymbol{n}(z)=\left(n_{1}(z), \ldots, n_{r}(z)\right)$.

Fix arbitrary sequences of non-negative integers $\boldsymbol{n}(z), \boldsymbol{n}(\infty)$ so that they satisfy relation (2.5). Let $\Lambda(z), \Lambda(\infty)$ be $s l_{r+1}$ integral dominant weights defined by the conditions

$$
\left(\Lambda(z), \alpha_{i}\right)=n_{i}(z), \quad\left(\Lambda(\infty), \alpha_{i}\right)=n_{i}(\infty) \quad(i=1, \ldots, r) .
$$

Then it is known, see [EH], MV1, that the number of spaces $V$ with the given $\boldsymbol{n}(z), \boldsymbol{n}(\infty), l$ is finite and bounded from above by the multiplicity of the module $L_{\Lambda(\infty)}$ in the tensor product $\otimes_{z} L_{\Lambda(z)}$. Moreover the number of such spaces is nonzero if and only if the above multiplicity is non-zero.

In this paper we study spaces of polynomials which have only two singular points $z_{1}=0$ and $z_{2}=1$. Moreover, we consider spaces only with special exponents at 1 . Namely, we restrict our attention to two cases:

$$
\boldsymbol{n}(1)=(k, 0, \ldots, 0) \quad \text { and } \quad \boldsymbol{n}^{\vee}(1)=(0, \ldots, 0, k) .
$$

For our purposes we parameterize the exponents with the above restriction using the parameters of Section 2.1. Given parameters $\boldsymbol{m}, \boldsymbol{l}, k$ we define two sets of parameters $\boldsymbol{n}(0), \boldsymbol{n}(1), l, \boldsymbol{n}(\infty)$ and $\boldsymbol{n}^{\vee}(0), \boldsymbol{n}^{\vee}(1), l^{\vee}, \boldsymbol{n}^{\vee}(\infty)$ by the formulas:

$$
\begin{aligned}
& n_{i}(1)=\delta_{1 i} k, \quad n_{i}(0)=m_{i}, \\
& l=l_{1}, \quad n_{i}(\infty)=l_{i-1}+l_{i+1}-2 l_{i}+m_{i}+\delta_{1 i} k \quad(i=1, \ldots, r)
\end{aligned}
$$

and

$$
\begin{array}{lll}
n_{i}^{\vee}(1)=\delta_{i r} k, & n_{i}^{\vee}(0) & =m_{r+1-i}, \\
l^{\vee}=l_{r}, & n_{i}^{\vee}(\infty) & =l_{r-i}+l_{r+2-i}-2 l_{r+1-i}+m_{r+1-i}+\delta_{r i} k \quad(i=1, \ldots, r) .
\end{array}
$$

Both $\boldsymbol{n}(0), \boldsymbol{n}(1), l, \boldsymbol{n}(\infty)$ and $\boldsymbol{n}^{\vee}(0), \boldsymbol{n}^{\vee}(1), l^{\vee}, \boldsymbol{n}^{\vee}(\infty)$ satisfy relation (2.5). 
In this parameterization the sets of exponents at infinity given by (2.4) take the form $\left\{e_{0}(\boldsymbol{m}, \boldsymbol{l}, k), \ldots, e_{r}(\boldsymbol{m}, \boldsymbol{l}, k)\right\}$ and $\left\{e_{0}^{\vee}(\boldsymbol{m}, \boldsymbol{l}, k), \ldots, e_{r}^{\vee}(\boldsymbol{m}, \boldsymbol{l}, k)\right\}$, where

$$
\begin{aligned}
& e_{i}(\boldsymbol{m}, \boldsymbol{l}, k)=k+\sum_{s=1}^{i} m_{s}-l_{i}+l_{i+1}+i, \\
& e_{i}^{\vee}(\boldsymbol{m}, \boldsymbol{l}, k)=\sum_{s=r+1-i}^{r} m_{s}-l_{r-i+1}+l_{r-i}+i,
\end{aligned}
$$

where $i=0, \ldots, r$. Note that for $0 \leq i \leq j \leq r$ we have

$$
e_{j}(\boldsymbol{m}, \boldsymbol{l}, k)-e_{i}(\boldsymbol{m}, \boldsymbol{l}, k)=e_{r-i}^{\vee}(\boldsymbol{m}, \boldsymbol{l}, k)-e_{r-j}^{\vee}(\boldsymbol{m}, \boldsymbol{l}, k) .
$$

Specializing the general statements to our case we obtain:

Lemma 2.2. If parameters $\boldsymbol{m}, \boldsymbol{l}, k$ are consistent, then there exists a unique space of polynomials $V(\boldsymbol{m}, \boldsymbol{l}, k)$ of dimension $r+1$ with exactly two singular points 0 and 1 and exponents given by (2.3) and (2.4) where $\boldsymbol{n}(0), \boldsymbol{n}(1), \boldsymbol{n}(\infty)$ are given by (2.6). If parameters $\boldsymbol{m}, \boldsymbol{l}, k$ are not consistent, then there exists no such a space.

Lemma 2.3. If parameters $\boldsymbol{m}, \boldsymbol{l}, k$ are consistent, then there exists a unique space of polynomials $U(\boldsymbol{m}, \boldsymbol{l}, k)$ of dimension $r+1$ with exactly two singular points 0 and 1 and exponents given by (2.3) and (2.4) where $\boldsymbol{n}(0), \boldsymbol{n}(1), \boldsymbol{n}(\infty)$ are equal to $\boldsymbol{n}^{\vee}(0), \boldsymbol{n}^{\vee}(1), \boldsymbol{n}^{\vee}(\infty)$ given by (2.6). If parameters $\boldsymbol{m}, \boldsymbol{l}, k$ are not consistent, then there exists no such a space.

Our parameters $\boldsymbol{m}$ are often fixed. We often drop the dependence on $\boldsymbol{m}$ from our notation and simply write $V(\boldsymbol{l}, k), U(\boldsymbol{l}, k)$.

2.3. Connection between spaces $V(\boldsymbol{m}, \boldsymbol{l}, k)$ and $U(\boldsymbol{m}, \boldsymbol{l}, k)$. Let $\boldsymbol{m}, \boldsymbol{l}, k$ be consistent parameters, $V=V(\boldsymbol{m}, \boldsymbol{l}, k)$ and $U=U(\boldsymbol{m}, \boldsymbol{l}, k)$ the corresponding spaces of polynomials.

We define an $r$-tuple of polynomials $\boldsymbol{T}=\left(T_{1}, \ldots, T_{r}\right)$ by

$$
T_{1}=(x-1)^{k} x^{m_{1}}, \quad T_{i}=x^{m_{i}} \quad(i=2, \ldots, r) .
$$

Lemma 2.4. We have

$$
W(V)=T_{1}^{r} T_{2}^{r-1} \ldots T_{r}, \quad W(U)=T_{r}^{r} T_{r-1}^{r-1} \ldots T_{1} .
$$

For $f_{1}, \ldots, f_{i} \in V$ and $g_{1}, \ldots, g_{i} \in U$ define the divided Wronskians

$$
\begin{aligned}
& W_{V}^{\dagger}\left(f_{1}, \ldots, f_{i}\right)=W\left(f_{1}, \ldots, f_{i}\right) T_{1}^{1-i} T_{2}^{2-i} \ldots T_{i-1}^{-1}, \\
& W_{U}^{\dagger}\left(g_{1}, \ldots, g_{i}\right)=W\left(g_{1}, \ldots, g_{i}\right) T_{r}^{1-i} T_{r-1}^{2-i} \ldots T_{r-i+2}^{-1} .
\end{aligned}
$$

We also define the divided Wronskian $W_{V}^{\dagger}\left(V_{1}\right)$ (resp. $\left.W_{U}^{\dagger}\left(U_{1}\right)\right)$ of any subspace $V_{1} \subset V$ (resp. $\left.U_{1} \subset U\right)$ as the divided Wronskian of any basis in $V_{1}$ (resp. $U_{1}$ ). The divided Wronskians $W_{V}^{\dagger}\left(V_{1}\right), W_{U}^{\dagger}\left(U_{1}\right)$ are defined up to multiplication by a non-zero constant.

The divided Wronskians are polynomials.

Lemma 2.5. We have

$$
\begin{aligned}
& V=\left\{W_{U}^{\dagger}\left(g_{1}, \ldots, g_{r}\right), g_{1}, \ldots, g_{r} \in U\right\}, \\
& U=\left\{W_{V}^{\dagger}\left(f_{1}, \ldots, f_{r}\right), f_{1}, \ldots, f_{r} \in U\right\} .
\end{aligned}
$$


Proof. The vector spaces $V$ and $\left\{W_{U}^{\dagger}\left(g_{1}, \ldots, g_{r}\right), g_{1}, \ldots, g_{r} \in U\right\}$ have the same dimensions, the same singular points, and exponents; see MV1 for details. Hence they coincide by Lemma 2.2.

2.4. Examples of spaces. Let $m_{1}, \ldots, m_{r}$ be non-negative numbers. Let $\mathbf{0}=$ $(0, \ldots, 0)$. Then the parameters $\boldsymbol{m}, \mathbf{0}, k=0$ are consistent.

Consider the space $V_{0}=V(\boldsymbol{m}, \mathbf{0}, 0)$. Let $e_{i}=e_{i}(\boldsymbol{m}, \mathbf{0}, 0)$. Then we have

$$
e_{i}=i+\sum_{j=1}^{i} m_{j}
$$

where $i=0, \ldots, r$; see (2.8).

Lemma 2.6. We have

$$
V_{0}=\operatorname{span}\left\{1=x^{e_{0}}, x^{e_{1}}, \ldots, x^{e_{r}}\right\} .
$$

Proof. The exponents of the space $V_{0}$ at 0 and infinity coincide. Thus the polynomial in $V_{0}$ with the highest order of zero at 0 has to be $x^{e_{r}}$. Similarly, a polynomial in $V_{0}$ with the order of zero at 0 equal to $e_{r-1}$ has to be a linear combination of $x^{e_{r-1}}$ and $x^{e_{r}}$. Continuing this argument we obtain the lemma.

Fix a non-negative integer $l$, and let $l^{0}=(l, \ldots, l)$. Assume that $m_{r} \geq l$. Then the parameters $\boldsymbol{m}, \boldsymbol{l}^{0}, k=l$ are consistent.

Consider the space $U_{l}=U\left(\boldsymbol{m}, \boldsymbol{l}^{0}, l\right)$. Let $e_{i}^{\vee}=e_{i}^{\vee}\left(\boldsymbol{m}, \boldsymbol{l}^{0}, l\right)$. Then we have

$$
e_{0}^{\vee}=l, \quad e_{i}^{\vee}=e_{i}^{\vee}\left(\boldsymbol{m}, \boldsymbol{l}^{0}, l\right)=i+\sum_{j=1}^{i} m_{r+1-j},
$$

where $i=1, \ldots, r$; see (2.9).

Lemma 2.7. There exists a unique monic polynomial $u_{0}(l)$ of degree $l$ such that

$$
U_{l}=\operatorname{span}\left\{u_{0}(l), x^{e_{1}^{\vee}}, \ldots, x^{e_{r}^{\vee}}\right\} .
$$

Moreover, $u_{0}(l)=x^{l}-B_{l} x^{l-1}+\ldots$, where

$$
B_{l}=l \prod_{s=1}^{r} \frac{e_{0}^{\vee}-e_{s}^{\vee}}{e_{0}^{\vee}-e_{s}^{\vee}-1}
$$

Proof. The last $r$ exponents of $U_{l}$ at 0 are equal to the last $r$ exponents of $U_{l}$ at infinity. Thus the functions $x^{e_{1}^{\vee}}, \ldots, x^{e_{r}^{\vee}}$ belong to $U_{l}$. Therefore there exists a unique monic polynomial $u_{0}(l)$ of degree $l$ such that the functions $u_{0}(l), x^{e_{1}^{\vee}}, \ldots, x^{e_{r}^{\vee}}$ form a basis of $U_{l}$. Moreover, we have

$$
W\left(u_{l}, x^{e_{1}^{\vee}}, \ldots, x^{e_{r}^{\vee}}\right)=c(x-1)^{l} x^{\sum_{s} e_{s}^{\vee}-(r+1) r / 2},
$$

where $c$ is a constant. The first non-trivial coefficient $B_{l}$ of the polynomial $u_{0}(l)$ is easily computed from this formula.

Corollary 2.8. We have

$$
U_{0}=\operatorname{span}\left\{1=x^{e_{0}^{\vee}(\boldsymbol{m}, \mathbf{0}, 0)}, x^{e_{1}^{\vee}(\boldsymbol{m}, \mathbf{0}, 0)}, \ldots, x^{e_{r}^{\vee}(\boldsymbol{m}, \mathbf{0}, 0)}\right\} .
$$

\section{RECURSION FOR SPACES OF POLYNOMIALS}

In this section we explain how the spaces of polynomials with different values of parameters are related to each other. 
3.1. Recursion of the first type. Fix a sequence $\boldsymbol{m}$ of non-negative integers. Let $\boldsymbol{l}, k$ be such that $\boldsymbol{m}, \boldsymbol{l}, k$ are consistent parameters.

Introduce linear first order differential operators

$$
D_{i}(\boldsymbol{l}, k)=x(x-1) \frac{d}{d x}-\left(k+\sum_{s=1}^{i} m_{s}-l_{i}+l_{i+1}+i\right)(x-1)-k-1,
$$

where $i=0,1, \ldots, r$. Note that the coefficients of $(x-1)$ are exactly the degrees of polynomials occurring in the space $V(\boldsymbol{l}, k)$. Namely, the coefficient of $(x-1)$ in $D_{i}(\boldsymbol{l}, k)$ is equal to $e_{i}(\boldsymbol{l}, k)$; see (2.8).

The operators $D_{i}(\boldsymbol{l}, k)$ are linearly dependent. Let

$$
A_{a b}=A_{a b}(\boldsymbol{l}, k)=e_{a}(\boldsymbol{l}, k)-e_{b}(\boldsymbol{l}, k) .
$$

Lemma 3.1. The vector space spanned by the operators $D_{i}(\boldsymbol{l}, k), i=0, \ldots, r$, has dimension two. Moreover, for $0 \leq i<j<s \leq r$, we have

$$
A_{j s} D_{i}(\boldsymbol{l}, k)+A_{s i} D_{j}(\boldsymbol{l}, k)+A_{i j} D_{s}(\boldsymbol{l}, k)=0 .
$$

Set $\mathbf{1}_{i}=(1, \ldots, 1,0, \ldots, 0)$ where we have $i$ ones and $r+1-i$ zeroes.

The operators $D_{i}$ possess the following holonomy property.

Lemma 3.2. For all $i, j \in\{0,1, \ldots, r\}$ we have

$$
D_{j}\left(\boldsymbol{l}+\mathbf{1}_{i}, k+1\right) D_{i}(\boldsymbol{l}, k)=D_{i}\left(\boldsymbol{l}+\mathbf{1}_{j}, k+1\right) D_{j}(\boldsymbol{l}, k) .
$$

Proof. It is an easy explicit check.

We give a conceptual reason for Lemma 3.2 in Section 3.3 ,

Theorem 3.3. Suppose $\boldsymbol{m}, \boldsymbol{l}, k$ and $\boldsymbol{m}, \boldsymbol{l}+\mathbf{1}_{i}, k+1$ are consistent parameters. Then operator $D_{i}(\boldsymbol{l}, k)$ maps $V(\boldsymbol{l}, k)$ to $V\left(\boldsymbol{l}+\mathbf{1}_{i}, k+1\right)$ and

$$
D_{i}(\boldsymbol{l}, k): V(\boldsymbol{l}, k) \rightarrow V\left(\boldsymbol{l}+\mathbf{1}_{i}, k+1\right)
$$

is an isomorphism of vector spaces.

Proof. We have

$$
\text { Ker } D_{j}(\boldsymbol{l}, k)=c(x-1)^{k+1} x^{\sum_{s=1}^{j} m_{s}-l_{j}+l_{j+1}+j-1},
$$

where $j=1, \ldots, r$ and $c$ is an arbitrary constant.

Note that the kernel of $D_{0}$ contains no non-trivial polynomial. Recall that the numbers $\sum_{s=1}^{i-1} m_{s}+i-1$ and $\sum_{s=1}^{i} m_{s}+i$ are consecutive orders of zeros at $x=0$ which appear in $V(\boldsymbol{l}, k)$. Under our assumptions we have

$$
\sum_{s=1}^{i-1} m_{s}+i-1<\sum_{s=1}^{i} m_{s}-l_{i}+l_{i+1}+i-1<\sum_{s=1}^{i} m_{s}+i .
$$

Therefore the intersection of $\operatorname{Ker} D_{i}(\boldsymbol{l}, k)$ and $V(\boldsymbol{l}, k)$ is trivial, because the orders of zero at $x=0$ of functions in these two spaces do not match.

It follows that $\bar{V}:=D_{i}(\boldsymbol{l}, k)(V(\boldsymbol{l}, k))$ is a space of polynomials of dimension $r+1$. Let us consider the exponents of $\bar{V}$.

Let $f_{1}, \ldots, f_{r+1}$ be a basis in $V(\boldsymbol{l}, k)$ compatible with the flag at infinity. We have $\operatorname{deg} f_{s}=e_{s}$, where $e_{s}$ are given by (2.8). Then we obviously have

$$
\begin{aligned}
& \operatorname{deg} D_{i} f_{s}=\operatorname{deg} f_{s}+1 \quad(s \neq i), \\
& \operatorname{deg} D_{i} f_{i} \leq \operatorname{deg} f_{i} .
\end{aligned}
$$


Also if $g \in V$ and $g(1)=0$, then the order of vanishing at $x=1$ of $D_{i}(\boldsymbol{l}, k) g(0)$ is at least $k+2$.

It follows from relation (2.5) that the above inequality is an equality and the exponents of $\bar{V}$ coincide with the exponents of $V\left(\boldsymbol{l}+\mathbf{1}_{i}, k+1\right)$. Therefore those two spaces coincide by the uniqueness part of Lemma 2.2 .

Corollary 3.4. Let $\boldsymbol{m}, \boldsymbol{l}, k$ be consistent and $\boldsymbol{m}, \boldsymbol{l}+\mathbf{1}_{i}, k+1$ are not consistent. Then $l_{i}-l_{i+1}=m_{i}$ and

$$
(x-1)^{k+1} x^{\sum_{s=1}^{i} m_{s}-l_{i}+l_{i+1}+i-1} \in V(\boldsymbol{l}, k) .
$$

In particular $\operatorname{dim} D_{i}(\boldsymbol{l}, k) V(\boldsymbol{l}, k)=r$.

Proof. If the statement of the lemma were wrong, then by the same argument as in the proof of Theorem 3.3. the space $D_{i}(\boldsymbol{l}, k) V(\boldsymbol{l}, k)$ would be a space of polynomials $V\left(\boldsymbol{l}+\mathbf{1}_{i}, k+1\right)$, which does not exist by Lemma 2.2 .

Lemma 3.5. Let $\boldsymbol{m}, \boldsymbol{l}, k$ be consistent parameters.

- There exist a sequence of indices $i(1), \ldots, i(k) \in\{0, \ldots, r\}$ such that $\boldsymbol{l}=$ $\sum_{s=1}^{k} \mathbf{1}_{i(s)}$ and $\boldsymbol{m}, \sum_{s=1}^{j} \mathbf{1}_{i(s)}, j$ are consistent parameters for $j=0, \ldots, k$.

- This sequence is unique up to permutation of its elements.

- 0 occurs $k-l_{1}$ times and $i$ occurs $l_{i}-l_{i+1}$ times $(i=1, \ldots, r)$.

Proof. Note that the addition of $\mathbf{1}_{i}$ adds one to $l_{i}-l_{i+1}$ and leaves all other differences $l_{j}-l_{j+1}, j \neq i$ intact. In particular the addition of $\mathbf{1}_{0}$ does not change either of these differences. The lemma follows.

Corollary 3.6. Let $\boldsymbol{l}, k$ be such that $\boldsymbol{m}, \boldsymbol{l}, k$ are consistent. Then there exists a unique linear differential operator $D_{l, k}$ of order $k$ and of the form

$$
D_{l, k}:=D_{j(k)}\left(\sum_{s=1}^{k-1} \mathbf{1}_{j(s)}, k-1\right) \ldots D_{j(2)}\left(\mathbf{1}_{j(1)}, 1\right) D_{j(1)}(\mathbf{0}, 0)
$$

with $j(s) \in\{0, \ldots, r\}, \sum_{s=1}^{k} \mathbf{1}_{j(s)}=\boldsymbol{l}$, such that $V(\boldsymbol{l}, k)=D_{\boldsymbol{l}, k} V(\mathbf{0}, 0)$.

Proof. The existence follows immediately from Theorem 3.3 and Lemma 3.5. The uniqueness follows from Lemma 3.5 and Corollary 3.4

Sometimes we stress the dependence of the operator $D_{l, k}$ on $\boldsymbol{m}$ and write $D_{\boldsymbol{m}, \boldsymbol{l}, k}$. We give some remarks about a basis in $V(\boldsymbol{l}, k)$.

Lemma 3.7. The space $V(\boldsymbol{l}, k)$ has a basis $\left\{\tilde{v}_{0}, \tilde{v}_{1}, \ldots, \tilde{v}_{r}\right\}$ such that $v_{0}$ is a polynomial of degree $l_{1}$ and $\tilde{v}_{i}, i=1, \ldots, r$, have the form

$$
\tilde{v}_{i}=(x-1)^{k+1} x^{\sum_{j=1}^{i-1} m_{j}+i-1} \sum_{j=0}^{m_{i}-l_{i}+l_{i+1}} a_{i j} x^{j},
$$

where $a_{i j}$ are non-zero constants.

Proof. The lemma is true for $V_{0}$,

$$
x^{e_{i}}-x^{e_{i-1}}=(x-1) x^{\sum_{j=1}^{i-1} m_{j}+i-1} \sum_{j=0}^{m_{i}} x^{j} .
$$


In addition if $D=x(x-1) d / d x+A(x-1)-(k+1)$, then

$$
D:(x-1)^{k+1} x^{a} \mapsto(k+1+a+A)(x-1)^{k+2} x^{a} .
$$

These two statements imply the lemma.

Note that using (3.4), one can write the constants $a_{i j}$ explicitly in a factored form.

Note also that Corollary 3.4 follows from Lemma 3.7

3.2. Recursion of the second type. The construction of this section is parallel to that of Section 3.1 .

Fix a sequence $\boldsymbol{m}$ of non-negative integers. Let $\boldsymbol{l}, k$ be such that $\boldsymbol{m}, \boldsymbol{l}, k$ are consistent parameters. Introduce linear first order differential operators

$$
D_{i}^{\vee}(\boldsymbol{l}, k)=x \frac{d}{d x}-\left(\sum_{s=i+1}^{r} m_{s}-l_{i+1}+l_{i}+r-i\right),
$$

where $i=0, \ldots, r$. Note that the constant coefficients in operators $D_{i}^{\vee}(\boldsymbol{l}, k)$ are exactly the degrees of polynomials occurring in the space $U(\boldsymbol{l}, k)$. Namely, the constant coefficient in $D_{i}^{\vee}(\boldsymbol{l}, k)$ is exactly $e_{r-i}^{\vee}(\boldsymbol{l}, k)$; see (2.9). (3.2).

The operators $D_{i}^{\vee}$ are linearly dependent. Recall the constants $A_{a b}$ given by

Lemma 3.8. The vector space spanned by operators $D_{i}^{\vee}(\boldsymbol{l}, k), i=0, \ldots, r$, has dimension two. Moreover, for $0 \leq i<j<s \leq r$, we have

$$
A_{j s} D_{i}^{\vee}(\boldsymbol{l}, k)+A_{s i} D_{j}^{\vee}(\boldsymbol{l}, k)+A_{i j} D_{s}^{\vee}(\boldsymbol{l}, k)=0 .
$$

The operators $D_{i}^{\vee}$ possess the following holonomy property.

Lemma 3.9. For all $i, j \in\{0,1, \ldots, r\}$ we have

$$
D_{j}^{\vee}\left(\boldsymbol{l}-\mathbf{1}_{i}, k-1\right) D_{i}^{\vee}(\boldsymbol{l}, k)=D_{i}^{\vee}\left(\boldsymbol{l}-\mathbf{1}_{j}, k-1\right) D_{j}^{\vee}(\boldsymbol{l}, k) .
$$

We give a conceptual reason for Lemma 3.9 in Section 3.3 .

Theorem 3.10. Suppose $\boldsymbol{m}, \boldsymbol{l}-\mathbf{1}_{i}, k-1$ and $\boldsymbol{m}, \boldsymbol{l}, k$ are consistent parameters. Then operator $D_{i}^{\vee}(\boldsymbol{l}, k)$ maps $U(\boldsymbol{l}, k)$ to $U\left(\boldsymbol{l}-\mathbf{1}_{i}, k-1\right)$ and

$$
D_{i}^{\vee}(\boldsymbol{l}, k): U(\boldsymbol{l}, k) \rightarrow U\left(\boldsymbol{l}-\mathbf{1}_{i}, k-1\right)
$$

is an isomorphism of vector spaces.

Proof. The proof is parallel to the proof of Theorem 3.3 .

Corollary 3.11. Let $\boldsymbol{l}, k$ be such that parameters $\boldsymbol{m}, \boldsymbol{l}, k$ are consistent. There exists a unique linear differential operator $D_{l, k}^{\vee}$ of order $k$ and of the form

$$
D_{l, k}^{\vee}:=D_{j(1)}^{\vee}\left(\mathbf{1}_{j(1)}, 1\right) D_{j(2)}^{\vee}\left(\mathbf{1}_{j(1)}+\mathbf{1}_{j(2)}, 2\right) \ldots D_{j(k)}^{\vee}\left(\sum_{s=1}^{k} \mathbf{1}_{j(s)}, k\right)
$$

with $j(s) \in\{0, \ldots, r\}, \sum_{s=1}^{k} \mathbf{1}_{j(s)}=\boldsymbol{l}$, such that $U(\mathbf{0}, 0)=D_{\boldsymbol{l}, k}^{\vee} U(\boldsymbol{l}, k)$. 
3.3. Canonical maps. Fix a sequence $\boldsymbol{m}$ of non-zero integers. Let $\boldsymbol{l}_{1}, k_{1}$ and $\boldsymbol{l}_{2}, k_{2}$ be such that the parameters $\boldsymbol{m}, \boldsymbol{l}_{1}, k_{1}$ and $\boldsymbol{m}, \boldsymbol{l}_{2}, k_{2}$ are consistent.

Recall that given any space of polynomials $V$ and $z \in \mathbb{C}$ we have the full flag of $V$ at $z, \mathcal{F}(V, z)=\left\{0 \subset F_{1}(V, z) \subset F_{2}(V, z) \subset \cdots \subset F_{r+1}(V, z)=V\right\}$, defined by the order of vanishing of polynomials in $V$ at $x=z$. We also have the full flag of $V$ at infinity, $\mathcal{F}(V, \infty)=\left\{0 \subset F_{1}(V, \infty) \subset F_{2}(V, \infty) \subset \cdots \subset F_{r+1}(V, \infty)=V\right\}$, defined by the degrees of polynomials in $V$.

We call a linear map $a: V\left(\boldsymbol{l}_{1}, k_{1}\right) \rightarrow V\left(\boldsymbol{l}_{2}, k_{2}\right)$ canonical if $a$ is an isomorphism and it is compatible with flags at singular points and infinity in the following sense:

$$
\begin{array}{ll}
a\left(F_{i}\left(V\left(\boldsymbol{l}_{1}, k_{1}\right), 0\right)\right)=F_{i}\left(V\left(\boldsymbol{l}_{2}, k_{2}\right), 0\right) & (i=1, \ldots, r), \\
a\left(F_{i}\left(V\left(\boldsymbol{l}_{1}, k_{1}\right), \infty\right)\right)=F_{i}\left(V\left(\boldsymbol{l}_{2}, k_{2}\right), \infty\right) & (i=1, \ldots, r), \\
a\left(F_{r}\left(V\left(\boldsymbol{l}_{1}, k_{1}\right), 1\right)\right)=F_{r}\left(V\left(\boldsymbol{l}_{2}, k_{2}\right), 1\right) . &
\end{array}
$$

We call a linear map $b: U\left(\boldsymbol{l}_{1}, k_{1}\right) \rightarrow U\left(\boldsymbol{l}_{2}, k_{2}\right)$ canonical if $b$ is an isomorphism and it is compatible with flags at singular points and infinity in the following sense:

$$
\begin{array}{ll}
b\left(F_{i}\left(U\left(\boldsymbol{l}_{1}, k_{1}\right), 0\right)\right)=F_{i}\left(U\left(\boldsymbol{l}_{2}, k_{2}\right), 0\right) & (i=1, \ldots, r), \\
b\left(F_{i}\left(U\left(\boldsymbol{l}_{1}, k_{1}\right), \infty\right)\right)=F_{i}\left(U\left(\boldsymbol{l}_{2}, k_{2}\right), \infty\right) & (i=1, \ldots, r), \\
b\left(F_{1}\left(U\left(\boldsymbol{l}_{1}, k_{1}\right), 1\right)\right)=F_{1}\left(U\left(\boldsymbol{l}_{2}, k_{2}\right), 1\right) . &
\end{array}
$$

Clearly the inverse of a canonical map is canonical, and a composition of canonical maps is canonical. A canonical map multiplied by a non-zero number is canonical.

Theorem 3.12. Let $\boldsymbol{m}, \boldsymbol{l}_{1}, k_{1}$ and $\boldsymbol{m}, \boldsymbol{l}_{2}, k_{2}$ be consistent parameters. Then there exist unique (up to multiplication by a non-zero number) canonical maps $V\left(\boldsymbol{l}_{1}, k_{1}\right) \rightarrow$ $V\left(\boldsymbol{l}_{2}, k_{2}\right)$ and $U\left(\boldsymbol{l}_{1}, k_{1}\right) \rightarrow U\left(\boldsymbol{l}_{2}, k_{2}\right)$.

Proof. We give a proof for the case of spaces $V(\boldsymbol{l}, k)$. The spaces $U(\boldsymbol{l}, k)$ are treated similarly. It is obviously sufficient to consider the case of $\left(\boldsymbol{l}_{1}, k_{1}\right)=(\mathbf{0}, 0)$, that is, the case of $V\left(\boldsymbol{l}_{1}, k_{1}\right)=V_{0}$.

We have a map $V_{0} \rightarrow V\left(\boldsymbol{l}_{2}, k_{2}\right)$ given by the operator $D_{\boldsymbol{l}_{2}, k_{2}}$; see Corollary 3.6. This map is clearly canonical; see the proof of Theorem 3.3.

Since we have the existence, to prove that the canonical map is unique it is sufficient to consider canonical maps $V_{0} \rightarrow V_{0}$.

Let $a: V_{0} \rightarrow V_{0}$ be a canonical map. Let $\mathcal{F}(0), \mathcal{F}(1), \mathcal{F}(\infty)$ be the corresponding flags at 0,1 and $\infty$. By degree reasons we have

$$
W_{V_{0}}^{\dagger}\left(F_{i}(\infty)\right)=1 \quad(i=1, \ldots, r)
$$

(up to non-zero constants).

For $i=1, \ldots, r+1$ consider $G_{i}=F_{i}(\infty) \cap F_{r+2-i}(0)$. By the definition of a canonical map, $a\left(G_{i}\right)=G_{i}$. But because of (3.6) we have $\operatorname{dim} G_{i}=1$. Let $G_{i}=c f_{i}, f_{i} \in V_{0}$. The basis $\left\{f_{1}, \ldots, f_{r+1}\right\}$ is compatible with $\mathcal{F}(\infty)$, and the basis $\left\{f_{r+1}, \ldots, f_{1}\right\}$ is compatible with $\mathcal{F}(0)$. In the basis $\left\{f_{1}, \ldots, f_{r+1}\right\}$ the map $a$ is diagonal. Let $a\left(f_{s}\right)=\mu_{s} f_{s}$.

Similarly for $i=1, \ldots, r+1$ we have $F_{i}(\infty) \cap F_{r+2-i}(1)=c g_{i}$ for some $g_{i} \in V_{0}$. The basis $\left\{g_{1}, \ldots, g_{r+1}\right\}$ is compatible with $\mathcal{F}(\infty)$ and the basis $\left\{g_{r+1}, \ldots, g_{1}\right\}$ is compatible with $\mathcal{F}(1)$. The span of $g_{2}, \ldots, g_{s}$ is the intersection of $F_{r}(1)$ and $F_{s}(\infty)$, and therefore it is preserved by the map $a$. 
Without loss of generality we can put $f_{1}=g_{1}=1$. It follows from Lemma 2.6 that without loss of generality we can assume

$$
f_{i}=x^{\sum_{s=1}^{i-1} m_{s}+i-1} .
$$

It follows that if we write $f_{i}=\sum_{s=1}^{i} \nu_{s i} g_{s}$, then $\nu_{i i} \neq 0$ and $\nu_{1 i} \neq 0$.

Since the map $a$ preserves the span of $g_{2}$ we get $\mu_{1}=\mu_{2}=\mu$. Since the map $a$ preserves the span of $g_{2}, g_{3}$ and this span does not contain $g_{1}$, we get $\mu_{3}=\mu$, and so on.

It follows that the map $a$ is scalar.

Note that the holonomy properties described in Lemmas 3.2 and 3.9 are natural to expect because of Theorem 3.12. since both compositions are the unique canonical maps when acting on our spaces of polynomials.

3.4. Inclusion property. Fix a sequence $\boldsymbol{m}$ of $r$ non-negative integers. Let a sequence $\boldsymbol{l}$ of $r$ non-negative integers and a non-negative integer $k$ be such that $\boldsymbol{m}, \boldsymbol{l}, k$ are consistent parameters. Let $s$ be a natural number. Let $\overline{\boldsymbol{l}}=\left(l_{1}, \ldots, l_{r}, 0, \ldots, 0\right)$ where we have $s$ zeroes, and $\overline{\boldsymbol{m}}=\left(m_{1}, \ldots, m_{r}, n_{1}, \ldots, n_{s}\right)$, where $n_{i}$ are nonnegative integers.

Lemma 3.13. We have

$$
V(\boldsymbol{m}, \boldsymbol{l}, k) \subset V(\overline{\boldsymbol{m}}, \overline{\boldsymbol{l}}, k) .
$$

Proof. The lemma is true for the case of $\boldsymbol{l}=0, k=0$; see Lemma2.6. The canonical isomorphisms $a: V(\boldsymbol{m}, \mathbf{0}, 0) \rightarrow V(\boldsymbol{m}, \boldsymbol{l}, k)$ and $\bar{a}: V(\overline{\boldsymbol{m}}, \overline{\mathbf{0}}, 0) \rightarrow V(\overline{\boldsymbol{m}}, \overline{\boldsymbol{l}}, k)$ are given by the same formula $a=D_{\boldsymbol{m}, \boldsymbol{l}, k}=D_{\overline{\boldsymbol{m}}, \bar{l}, k}=\bar{a}$. The lemma follows.

Canonical maps are compatible with the inclusion described in Lemma 3.13.

Namely, let $\boldsymbol{l}^{1}, k^{1}$ be such that $\boldsymbol{m}, \boldsymbol{l}^{1}, k^{1}$ are also consistent parameters. We set $\bar{l}^{1}=\left(l_{1}^{1}, \ldots, l_{r}^{1}, 0, \ldots, 0\right)$ where we have $s$ zeroes. We have canonical isomorphisms

$$
a: V(\boldsymbol{m}, \boldsymbol{l}, k) \rightarrow V\left(\boldsymbol{m}, \boldsymbol{l}^{1}, k^{1}\right), \quad \bar{a}: V(\overline{\boldsymbol{m}}, \overline{\boldsymbol{l}}, k) \rightarrow V\left(\overline{\boldsymbol{m}}, \overline{\boldsymbol{l}}^{1}, k^{1}\right),
$$

defined by

$$
a=D_{\boldsymbol{m}, \boldsymbol{l}^{1}, k^{1}} \circ\left(D_{\boldsymbol{m}, \boldsymbol{l}, k}\right)^{-1}, \quad \bar{a}=D_{{\overline{\boldsymbol{m}}, \bar{l}^{1}, k^{1}}^{1}} \circ\left(D_{\overline{\boldsymbol{m}}, \bar{l}, k}\right)^{-1} .
$$

Corollary 3.14. The canonical map $a$ is the restriction of the canonical map $\bar{a}$ to $V(\boldsymbol{m}, \boldsymbol{l}, k) \subset V(\overline{\boldsymbol{m}}, \overline{\boldsymbol{l}}, k)$.

Spaces $U(\boldsymbol{m}, \boldsymbol{l}, k)$ possess similar properties. For example, the analog of Lemma 3.13 for spaces $U(\boldsymbol{m}, \boldsymbol{l}, k)$ has the following form.

Lemma 3.15. We have the surjective well-defined map of vector spaces of polynomials

$$
\begin{aligned}
\pi: U(\overline{\boldsymbol{m}}, \overline{\boldsymbol{l}}, k) & \rightarrow U(\boldsymbol{m}, \boldsymbol{l}, k), \\
g & \mapsto x^{-n_{1}} \frac{d}{d x} \quad \ldots x^{-n_{s-1}} \frac{d}{d x} x^{-n_{s}} \frac{d}{d x} g .
\end{aligned}
$$


3.5. Duality. Fix a sequence $\boldsymbol{m}$ of non-negative integers. Let $\boldsymbol{l}, k$ be such that $\boldsymbol{m}, \boldsymbol{l}, \boldsymbol{k}$ are consistent parameters.

We use Lemma 2.5 to define a pairing $\langle,\rangle_{\boldsymbol{l}, k}: V(\boldsymbol{l}, k) \otimes U(\boldsymbol{l}, k) \rightarrow \mathbb{C}$ as follows. For $f \in V(\boldsymbol{l}, k), g \in U(\boldsymbol{l}, k)$ we set

$$
\langle f, g\rangle_{\boldsymbol{l}, k}=W_{V(\boldsymbol{l}, k)}^{\dagger}\left(f, f_{1}, \ldots, f_{r}\right) \quad \text { if } g=W_{V(\boldsymbol{l}, k)}^{\dagger}\left(f_{1}, \ldots, f_{r}\right) ;
$$

see Section 6.1 in MV1, where this pairing is studied in a more general setting.

Lemma 3.16. The pairing $\langle,\rangle_{\boldsymbol{l}, k}: V(\boldsymbol{l}, k) \otimes U(\boldsymbol{l}, k) \rightarrow \mathbb{C}$ is a well-defined bilinear, non-degenerate pairing.

Proof. Lemma 3.16 follows from Lemma 2.5.

Recall that the degrees of the polynomials $e_{i}$ and $e_{j}^{\vee}$ occurring in $V(\boldsymbol{l}, k)$ and $U(\boldsymbol{l}, k)$ are given by (2.8) and (2.9) .

Lemma 3.17. Let $f, g$ be monic polynomials such that $f \in V(\boldsymbol{l}, k), \operatorname{deg} f=e_{i}$, and $g \in U(\boldsymbol{l}, k), \operatorname{deg} g=e_{r-j}^{\vee}$. Then we have

$$
\langle f, g\rangle_{l, k}=\prod_{s=0,}^{r}\left(e_{s}-e_{i}\right) .
$$

Proof. For $a_{1} \leq \cdots \leq a_{j}$ we have the formula

$$
W\left(x^{a_{1}}, \ldots, x^{a_{j}}\right)=x^{\sum_{s=1}^{j} a_{s}-j(j+1) / 2} \prod_{s>t}\left(a_{s}-a_{t}\right) .
$$

We compute (denoting the terms of lower degrees by dots):

$$
\begin{aligned}
& \langle f, g\rangle_{\boldsymbol{l}, k}= \\
& W_{V(l, k)}^{\dagger}\left(f, x^{e_{0}}+\ldots, \ldots, x^{\left.e_{r-j}+\ldots, \ldots, x^{e_{r}}+\ldots\right)}\left(\prod_{\substack { s>>,{c}{s \neq r-j \\
t \neq r-j{ s > > , \\
\begin{subarray} { c } { s \neq r - j \\
t \neq r - j } }\end{subarray}}\left(e_{s}-e_{t}\right)\right)^{-1}\right. \\
& =(-1)^{i} \prod_{s>t}\left(e_{s}-e_{t}\right)\left(\prod_{s>t, s \neq i, t \neq i}\left(e_{s}-e_{t}\right)\right)^{-1}=\prod_{s=0, s \neq i}^{r}\left(e_{s}-e_{i}\right) .
\end{aligned}
$$

Proposition 3.18. The linear operators $D_{i}(\boldsymbol{l}, k): V(\boldsymbol{l}, k) \rightarrow V\left(\boldsymbol{l}+\mathbf{1}_{i}, k+1\right)$ and $-D_{i}^{\vee}\left(\boldsymbol{l}+\mathbf{1}_{i}, k+1\right): U\left(\boldsymbol{l}+\mathbf{1}_{i}, k+1\right) \rightarrow U(\boldsymbol{l}, k)$ are adjoint. Namely, for any $f \in V(\boldsymbol{l}, k)$ and any $g \in U(\boldsymbol{l}, k)$ we have

$$
\left\langle D_{i}(\boldsymbol{l}, k) f, g\right\rangle_{\boldsymbol{l}_{+\mathbf{1}_{i}, k+1}}=-\left\langle f, D_{i}^{\vee}\left(\boldsymbol{l}+\mathbf{1}_{i}, k+1\right) g\right\rangle_{\boldsymbol{l}, k} .
$$

Proof. The operator $D_{i}(\boldsymbol{l}, k)$ defines a canonical map. It follows from definitions that the map adjoint to a canonical map is canonical. Therefore, by the uniqueness of the canonical map, the map defined by $D_{i}^{\vee}\left(\boldsymbol{l}+\mathbf{1}_{i}, k+1\right)$ is a constant multiple of the map adjoint to the one defined by $D_{i}(\boldsymbol{l}, k)$.

We claim the constant is -1 . Let $f_{0}, \ldots, f_{r}$ be a basis of monic polynomials in $V(\boldsymbol{l}, k)$ compatible with the flag at infinity. We have $\operatorname{deg} f_{s}=e_{s}$ and $f_{i}$ are monic. Let $\tilde{e}_{s}=e_{s}+1-\delta_{i, s}$ be the degrees of the polynomials in $V\left(\boldsymbol{l}+\mathbf{1}_{i}, k+1\right)$. 
Let $g \in U\left(\boldsymbol{l}+\mathbf{1}_{i}, k+1\right)$ be a monic polynomial of maximal degree. In the next calculation we assume that $i>0$; the case of $i=0$ is similar. By Lemma 3.17.

$$
\begin{aligned}
\left\langle D_{i}(\boldsymbol{l}, k) f_{0}, g\right\rangle & =\left(e_{0}-e_{i}\right)\left\langle x^{l_{1}+1}+\ldots, g\right\rangle \\
& =\left(e_{0}-e_{i}\right) \prod_{s, s \neq 0}\left(\tilde{e}_{s}-\tilde{e}_{0}\right)=\left(e_{0}-e_{i}+1\right) \prod_{s=1}^{r}\left(e_{s}-e_{0}\right) .
\end{aligned}
$$

Here we denoted the terms of lower degrees by dots. Similarly we obtain

$$
\left\langle f_{0}, D_{i}^{\vee}\left(\boldsymbol{l}+\mathbf{1}_{i}, k+1\right) g\right\rangle=\left(e_{r}^{\vee}-e_{r-i}^{\vee}-1\right) \prod_{s=1}^{r}\left(e_{s}-e_{0}\right) .
$$

Therefore by (2.10) we have $\left\langle D_{i}(\boldsymbol{l}, k) f_{0}, g\right\rangle=-\left\langle f_{0}, D_{i}^{\vee}\left(\boldsymbol{l}+\mathbf{1}_{i}, k+1\right) g\right\rangle$ and the constant is -1 .

\section{SPaCES $V(\boldsymbol{m}, \boldsymbol{l}, k)$ AND JaCOBI-PiÑeIRo POlynomials}

In this section we present an explicit basis of spaces $V(\boldsymbol{m}, \boldsymbol{l}, k)$ in terms of multiple orthogonal polynomials, called Jacobi-Piñeiro polynomials.

4.1. Rodrigues formula. Let $k, l_{1}, \ldots, l_{r}$ be non-negative integers such that $k \geq$ $l_{1} \geq \cdots \geq l_{r}$. Let $m_{1}, \ldots, m_{r}$ be generic complex numbers.

For $i=0, \ldots, r$ we define a differential operator which acts on functions of $x$ :

$$
\tilde{D}_{i}(\boldsymbol{m}, \boldsymbol{l}, k)=x^{\sum_{j=1}^{i} m_{j}+i} \frac{d^{l_{i}-l_{i+1}}}{d x^{l_{i}-l_{i+1}}} x^{l_{i}-l_{i+1}-\sum_{j=1}^{i} m_{j}-i} .
$$

This operator is the composition of multiplication by a monomial, multiple differentiation, and another multiplication by a monomial.

Lemma 4.1. The differential operators $\tilde{D}_{i}(\boldsymbol{m}, \boldsymbol{l}, k)$ commute for all values of the parameters:

$$
\tilde{D}_{i}\left(\boldsymbol{m}_{1}, \boldsymbol{l}_{1}, k_{1}\right) \tilde{D}_{j}\left(\boldsymbol{m}_{2}, \boldsymbol{l}_{2}, k_{2}\right)=\tilde{D}_{j}\left(\boldsymbol{m}_{2}, \boldsymbol{l}_{2}, k_{2}\right) \tilde{D}_{i}\left(\boldsymbol{m}_{1}, \boldsymbol{l}_{1}, k_{1}\right) .
$$

Set

$$
\omega(x):=(x-1)^{-k-1} x^{-\sum_{i=1}^{r} m_{i}-r} .
$$
0 .

Recall the operator $D_{\boldsymbol{m}, \boldsymbol{l}, k}$ given by (3.3) and our conventions $l_{0}=k$ and $l_{r+1}=$

Proposition 4.2. We have the equality of differential operators

$$
\begin{aligned}
D_{\boldsymbol{m}, \boldsymbol{l}, k} & =(x-1)^{k+1} \tilde{D}_{r}(\boldsymbol{m}, \boldsymbol{l}, k) \tilde{D}_{r-1}(\boldsymbol{m}, \boldsymbol{l}, k) \ldots \tilde{D}_{0}(\boldsymbol{m}, \boldsymbol{l}, k) \frac{1}{x-1} \\
& =\omega^{-1}(x) \frac{d^{l_{r}-l_{r+1}}}{d x^{l_{r}-l_{r+1}}} x^{l_{r}-l_{r+1}-m_{r}-1} \frac{d^{l_{r-1}-l_{r}}}{d x^{l_{r+1}-l_{r}}} x^{l_{r-1}-l_{r}-m_{r-1}-1} \ldots \\
x^{l_{1}-l_{2}-m_{1}-1} & \frac{d^{l_{0}-l_{1}}}{d x^{l_{0}-l_{1}}} \frac{x^{l_{0}-l_{1}}}{x-1} .
\end{aligned}
$$

Proof. For any complex $a, b$ and non-negative integer $s$ we have the following identity of differential operators:

$$
\begin{aligned}
& \left(x(x-1) \frac{d}{d x}-a(x-1)-b-s\right)\left(x(x-1) \frac{d}{d x}-a(x-1)-b-s+1\right) \ldots \\
& \ldots\left(x(x-1) \frac{d}{d x}-a(x-1)-b\right)=(x-1)^{b+s+1} x^{a-b+1} \frac{d^{s+1}}{d x^{s+1}}(x-1)^{-b} x^{-a+b+s} .
\end{aligned}
$$


Indeed, both operators are of order $s+1$ with the symbol $(x(x-1))^{s+1} d^{s+1}$. Moreover, it is easy to check that both operators kill the functions

$$
(x-1)^{b+i} x^{a-b-s} \quad(i=0, \ldots, s) .
$$

It follows that these two operators are the same.

The proposition follows from the above identity.

4.2. Weyl group action. Let $k, l_{1}, \ldots, l_{r}$ be non-negative integers satisfying the conditions $k \geq l_{1} \geq \cdots \geq l_{r}$. Let $m_{1}, \ldots, m_{r}$ be generic complex numbers.

Let $\mathcal{S}_{r+1}$ be the the Weyl group of $s l_{r+1}$. Then $\mathcal{S}_{r+1}$ is isomorphic to the group of permutations of the set $\{0,1 \ldots, r\}$. We denote by $w(i)$ the image of $i \in\{0,1 \ldots, r\}$ under permutation $w$. The group $\mathcal{S}_{r+1}$ is generated by simple transpositions $s_{1}, \ldots, s_{r}$. The simple transposition $s_{i}$ permutes elements $i$ and $i-1$ and preserves all other elements of the set $\{0,1, \ldots, r\}$.

The group $\mathcal{S}_{r+1}$ acts on $s l_{r+1}$ weights by the formula

$$
s_{i} \cdot \lambda=\lambda-\left(\lambda+\rho, \alpha_{i}\right) \alpha_{i} .
$$

The first formula in (2.1) identifies $s l_{r+1}$ weights with vectors $\boldsymbol{m}$. Under this identification the Weyl group action becomes:

$$
\mathbf{s}_{i} \cdot \boldsymbol{m}=\left(m_{1}, \ldots, m_{i-2}, m_{i-1}+m_{i}+1,-m_{i}-2, m_{i+1}+m_{i}+1, m_{i+2}, \ldots, m_{r}\right) .
$$

We denote the result of the action of " $i w \in \mathcal{S}_{r+1}$ on $\boldsymbol{m}$ by $\boldsymbol{w} \cdot \boldsymbol{m}$.

Given $k$, define another action of the Weyl group $\mathcal{S}_{r+1}$ on the sequences of numbers $\boldsymbol{l}$ by the formulas

$$
\left(s_{i}\right)_{k} \boldsymbol{l}=\left(l_{1}, \ldots, l_{i-1}, l_{i-1}+l_{i+1}-l_{i}, l_{i+1}, \ldots, l_{r}\right),
$$

where as always $l_{0}=k$ and $l_{r+1}=0$. We denote the result of the action of $w \in \mathcal{S}_{r+1}$ on $\boldsymbol{l}$ by $(w)_{k} \boldsymbol{l}$.

If we consider the map $\mathbb{C}^{r} \rightarrow \mathbb{C}^{r+1}$ mapping $\boldsymbol{l} \rightarrow\left\{l_{0}-l_{1}, l_{1}-l_{2}, \ldots, l_{r}-l_{r+1}\right\}$, then the second Weyl group action descends to the standard permutation action of coordinates.

The Weyl group acts on operators $\tilde{D}_{i}(\boldsymbol{m}, \boldsymbol{l}, k)$ as follows.

Lemma 4.3. We have

$$
\begin{aligned}
\tilde{D}_{i}\left(s_{1} \cdot \boldsymbol{m},\left(s_{1}\right)_{k} \boldsymbol{l}, k\right) & =x^{-m_{1}-1} \tilde{D}_{s_{1}(i)}(\boldsymbol{m}, \boldsymbol{l}, k) x^{m_{1}+1}, \\
\tilde{D}_{i}\left(s_{j} \cdot \boldsymbol{m},\left(s_{j}\right)_{k} \boldsymbol{l}, k\right) & =\tilde{D}_{s_{j}(i)}(\boldsymbol{m}, \boldsymbol{l}, k),
\end{aligned}
$$

where $j=2, \ldots, r$.

4.3. Basis in $V(\boldsymbol{m}, \boldsymbol{l}, k)$. Let $\boldsymbol{m}, \boldsymbol{l}, k$ be consistent parameters.

The following lemma follows from Theorem 3.3 and Lemma 2.6

Lemma 4.4. The space $V(\boldsymbol{m}, \boldsymbol{l}, k)$ has a basis of the form

$$
\left\{v_{0}(\boldsymbol{m}, \boldsymbol{l}, k), v_{1}(\boldsymbol{m}, \boldsymbol{l}, k) x^{m_{1}+1}, \ldots, v_{r}(\boldsymbol{m}, \boldsymbol{l}, k) x^{\sum_{i=1}^{r} m_{i}+r}\right\},
$$

where $v_{i}(\boldsymbol{m}, \boldsymbol{l}, k)$ is a monic polynomial of degree

$$
\operatorname{deg} v_{i}(\boldsymbol{m}, \boldsymbol{l}, k)=k-l_{i}+l_{i+1}
$$

whose coefficients are rational functions of $\boldsymbol{m}$ with coefficients in $\mathbb{Q}$. Moreover, the polynomial $v_{i}(\boldsymbol{m}, \boldsymbol{l}, k)$ is obtained via the action of the operator $D_{\boldsymbol{m}, \boldsymbol{l}, k}$ as follows:

$$
v_{i}(\boldsymbol{m}, \boldsymbol{l}, k)=x^{-\sum_{j=1}^{i} m_{j}-i} D_{\boldsymbol{m}, \boldsymbol{l}, k}\left(x^{\sum_{j=1}^{i} m_{j}+i}\right) .
$$


The polynomials $v_{i}$ are permuted by the Weyl group.

Proposition 4.5. Let $w \in \mathcal{S}_{r+1}$ be an element of the Weyl group. Then

$$
v_{i}\left(w \cdot \boldsymbol{m},(w)_{k} \boldsymbol{l}, k\right)=v_{w(i)}(\boldsymbol{m}, \boldsymbol{l}, k) .
$$

Proof. For $i=2, \ldots, r$ from Lemmas 4.3 and 4.1 we have the equality of operators

$$
D_{s_{i} \cdot \boldsymbol{m},\left(s_{i}\right)_{k} \boldsymbol{l}, k}=D_{\boldsymbol{m}, \boldsymbol{l}, k}
$$

and

$$
D_{s_{1} \cdot \boldsymbol{m},\left(s_{1}\right)_{k} \boldsymbol{l}, k}=x^{-m_{1}-1} D_{\boldsymbol{m}, \boldsymbol{l}, k} x^{m_{1}+1} .
$$

The proposition follows from formula (4.2).

Let $P(\boldsymbol{m}, \boldsymbol{l}, k)$ be the monic polynomial defined by the application of differential operators:

$$
\begin{aligned}
P(\boldsymbol{m}, \boldsymbol{l}, k)= & c(x-1)^{k+1} \tilde{D}_{r}(\boldsymbol{m}, \boldsymbol{l}, k) \tilde{D}_{r-1}(\boldsymbol{m}, \boldsymbol{l}, k) \ldots \tilde{D}_{1}(\boldsymbol{m}, \boldsymbol{l}, k)\left((x-1)^{l_{1}-k-1}\right) \\
= & c \omega^{-1}(x) \frac{d^{l_{r}-l_{r+1}}}{d x^{l_{r}-l_{r+1}}} x^{l_{r}-l_{r+1}-m_{r}-1} \frac{d^{l_{r-1}-l_{r}}}{d x^{l_{r+1}-l_{r}}} \ldots \\
& x^{l_{2}-l_{3}-m_{2}-1} \frac{d^{l_{1}-l_{2}}}{d x^{l_{1}-l_{2}}}\left(x^{l_{1}-l_{2}-m_{1}-1}(x-1)^{l_{1}-k-1}\right)
\end{aligned}
$$

where $c$ is a non-zero constant.

Following [ABV], we call $P(\boldsymbol{m}, \boldsymbol{l}, k)$ the Jacobi-Piñeiro polynomial. Clearly, $P(\boldsymbol{m}, \boldsymbol{l}, k)$ is a polynomial of degree $l_{1}$ in $x$, whose coefficients are rational functions in $\boldsymbol{m}, k$ with coefficients in $\mathbb{Q}$.

Sometimes we drop the dependence on $\boldsymbol{m}$ from our notation and simply write $P(\boldsymbol{l}, k)$.

Proposition 4.6. Let $w \in \mathcal{S}_{r+1}$ be an element of the Weyl group such that $w(0)=$ $i$. Then

$$
v_{i}(\boldsymbol{m}, \boldsymbol{l}, k)=P\left(w \cdot \boldsymbol{m},(w)_{k} \boldsymbol{l}, k\right) .
$$

Proof. In view of Lemma 4.5 it is sufficient to prove the proposition for $w=i d$.

We have the equality of functions

$$
\begin{aligned}
v_{0}(\boldsymbol{m}, l, k) & =D_{\boldsymbol{m}, l, k}(1) \\
& =(x-1)^{k+1} \tilde{D}_{r}(\boldsymbol{m}, \boldsymbol{l}, k) \tilde{D}_{r-1}(\boldsymbol{m}, \boldsymbol{l}, k) \ldots \tilde{D}_{0}(\boldsymbol{m}, \boldsymbol{l}, k)\left(\frac{1}{x-1}\right) .
\end{aligned}
$$

But

$$
D_{0}(\boldsymbol{m}, \boldsymbol{l}, k)\left(\frac{1}{x-1}\right)=\frac{d^{k-l_{1}}}{d x^{k-l_{1}}}\left(\frac{x^{k-l_{1}}}{x-1}\right)=\left(k-l_{1}\right) !(x-1)^{l_{1}-k-1} .
$$

The proposition follows.

Corollary 4.7. Let $w \in \mathcal{S}_{r+1}$ be such that $w(0)=0$. Then

$$
P(\boldsymbol{m}, \boldsymbol{l}, k)=P\left(w \cdot \boldsymbol{m},(w)_{k} \boldsymbol{l}, k\right) .
$$

We also note that the divided Wronskian of all elements of the basis is a constant, which gives an identity for Jacobi-Piñeiro polynomials. 
Corollary 4.8. We have

$W\left(P(\boldsymbol{m}, \boldsymbol{l}, k), P\left(w_{1} \cdot \boldsymbol{m},\left(w_{1}\right)_{k} \boldsymbol{l}, k\right) x^{m_{1}+1}, \ldots, P\left(w_{r} \cdot \boldsymbol{m},\left(w_{r}\right)_{k} \boldsymbol{l}, k\right) x^{\sum_{i=1}^{r} m_{i}+r}\right)$

$$
=\text { const } x^{\sum_{i=1}^{r}(r+1-i) m_{i}}(x-1)^{r k} \text {, }
$$

where $w_{i}=s_{i} \ldots s_{2} s_{1}$.

4.4. Properties of Jacobi-Piñeiro polynomials. Jacobi-Piñeiro polynomials are examples of multiple orthogonal polynomials; see $[\mathrm{P},[\mathrm{IN}], \mathrm{ABV}]$ and references therein. In this section we prove some properties of Jacobi-Piñeiro polynomials, which we need for applications.

We fix a sequence $\boldsymbol{m}$ of arbitrary complex numbers.

Define constants $A_{i}(\boldsymbol{l}, k)$ by

$$
\begin{aligned}
& A_{0}(\boldsymbol{l}, k)=\prod_{s=0}^{r} \frac{k+\sum_{i=1}^{s} m_{s}-l_{1}+s+1}{k+\sum_{i=1}^{s} m_{s}-l_{1}+s+1+l_{s+1}-l_{s}}, \\
& A_{i}(\boldsymbol{l}, k)=e_{0}(\boldsymbol{l}, k)-e_{i}(\boldsymbol{l}, k)=l_{1}-\sum_{s=1}^{i} m_{s}-k+l_{i}-l_{i+1}-i,
\end{aligned}
$$

where $i=1, \ldots, r$.

We have

$$
A_{0}(\boldsymbol{l}, k)=\left(k-l_{1}+1\right) \prod_{s=1}^{r} \frac{e_{s}(\boldsymbol{l}, k)-e_{0}(\boldsymbol{l}, k)+l_{s}-l_{s+1}+1}{e_{s}(\boldsymbol{l}, k)-e_{0}(\boldsymbol{l}, k)+1} .
$$

These numbers satisfy the following property.

Lemma 4.9. We have

$$
A_{i}\left(\boldsymbol{l}+\mathbf{1}_{j}, k+1\right) A_{j}(\boldsymbol{l}, k)=A_{j}\left(\boldsymbol{l}+\mathbf{1}_{i}, k+1\right) A_{i}(\boldsymbol{l}, k) .
$$

The recursion for spaces of polynomials translates to the recursion for the JacobiPiñeiro polynomials:

Proposition 4.10. We have

$$
D_{i}(\boldsymbol{l}, k) P(\boldsymbol{l}, k)=A_{i}(\boldsymbol{l}, k) P\left(\boldsymbol{l}+\mathbf{1}_{i}, k+1\right) .
$$

Proof. First we suppose that the parameters $\boldsymbol{m}, \boldsymbol{l}, k$ and $\boldsymbol{m}, \boldsymbol{l}+\mathbf{1}_{i}, k+1$ are consistent.

By Theorem 3.3 and Proposition 4.6 we obtain that $D_{i}(\boldsymbol{l}, k) P(\boldsymbol{l}, k)$ is a constant multiple of $P\left(\boldsymbol{l}+\mathbf{1}_{i}, k+1\right)$. Call these constants $\tilde{A}_{i}(\boldsymbol{l}, k)$.

Recall that $P(\boldsymbol{l}, k)$ is monic. Comparing the highest degree terms we obtain $\tilde{A}_{i}(\boldsymbol{l}, k)=A_{i}(\boldsymbol{l}, k)$ for all cases except for $i=0$ when the highest degree terms cancel and we need to consider terms of the next degree.

By Lemma 3.2 the numbers $\tilde{A}_{i}(\boldsymbol{l}, k)$ satisfy the relation of Lemma 4.9, We also have the initial conditions

$$
\tilde{A}_{0}(\mathbf{0}, k)=k+1 .
$$

The numbers $\tilde{A}_{0}(\boldsymbol{l}, k)$ are uniquely determined by $\tilde{A}_{i}(\boldsymbol{l}, k),(i=1, \ldots, r)$ and these properties. But numbers $A_{0}(\boldsymbol{l}, k)$ satisfy the same recursion. Therefore $\tilde{A}_{0}(\boldsymbol{l}, k)=\tilde{A}_{0}(\boldsymbol{l}, k)$.

The case of general parameters $\boldsymbol{m}, \boldsymbol{l}, k$ follows by analytic continuation with respect to $\boldsymbol{m}, k$. 
Lemma 3.1 and Proposition 4.10 immediately imply the following 3-term relation for Jacobi-Piñeiro polynomials.

Let $\boldsymbol{l}$ be a partition.

Corollary 4.11. We have

$A_{i} A_{j s} P\left(\boldsymbol{l}+\mathbf{1}_{i}, k+1\right)+A_{j} A_{i s} P\left(\boldsymbol{l}+\mathbf{1}_{j}, k+1\right)+A_{s} A_{i j} P\left(\boldsymbol{l}+\mathbf{1}_{s}, k+1\right)=0$, where $A_{t}=A_{t}(\boldsymbol{l}, k), A_{t p}=A_{t p}(\boldsymbol{l}, k)$.

\section{Spaces $U(\boldsymbol{m}, \boldsymbol{l}, k)$}

In this section we compute explicitly a basis in the space $U(\boldsymbol{m}, \boldsymbol{l}, k)$.

5.1. Basis in $U(\boldsymbol{m}, \boldsymbol{l}, k)$. Let $\boldsymbol{m}, \boldsymbol{l}, k$ be consistent parameters. Recall that we use the convention $l_{0}=k$.

The following lemma follows from Theorem 3.10 .

Lemma 5.1 ( $[\mathrm{Sc})$. The space $U(\boldsymbol{m}, \boldsymbol{l}, k)$ has a basis of the form

$$
\left\{u_{0}(\boldsymbol{m}, \boldsymbol{l}, k), u_{1}(\boldsymbol{m}, \boldsymbol{l}, k) x^{m_{r}+1}, \ldots, u_{r}(\boldsymbol{m}, \boldsymbol{l}, k) x^{\sum_{i=1}^{r} m_{i}+r}\right\},
$$

where $u_{i}(\boldsymbol{m}, \boldsymbol{l}, k)$ is a monic polynomial of degree

$$
\operatorname{deg} u_{i}(\boldsymbol{m}, \boldsymbol{l}, k)=l_{r-i}-l_{r-i+1}
$$

whose coefficients are rational functions of $\boldsymbol{m}, k$ with coefficients in $\mathbb{Q}$.

The polynomials $u_{i}$ are permuted by the Weyl group.

Lemma 5.2. Let $w \in \mathcal{S}_{r+1}$ be an element of the Weyl group. Then

$$
u_{r-i}\left(w \cdot \boldsymbol{m},(w)_{k} \boldsymbol{l}, k\right)=u_{r-w(i)}(\boldsymbol{m}, \boldsymbol{l}, k) .
$$

Proof. We have the formula

$$
u_{r-i}(\boldsymbol{m}, \boldsymbol{l}, k)=c_{i} W^{\dagger}\left(f_{0}, f_{1}, \ldots, \widehat{f}_{i}, \ldots, f_{r}\right) x^{-\sum_{s=r-i+1}^{r} m_{s}-i}
$$

where $c_{i}$ are non-zero constants, $f_{i}=v_{i}(\boldsymbol{m}, \boldsymbol{l}, k) x^{\sum_{s=1}^{i} m_{s}+i}$ and $v_{i}$ are as in Lemma 4.4. Now the lemma follows from Lemma 4.5.

Note that formula (5.1) determines $u_{i}$ in terms of Jacobi-Piñeiro polynomials.

5.2. Recursion and 3-term relation. Fix any sequence of $r$ complex numbers $\boldsymbol{m}$. Define constants $A_{i}^{\vee}(\boldsymbol{l}, k)$ by

$$
\begin{aligned}
& A_{i}^{\vee}(\boldsymbol{l}, k)=e_{0}^{\vee}(\boldsymbol{l}, k)-e_{r-i}^{\vee}(\boldsymbol{l}, k)=l_{r}-\sum_{s=i+1}^{r} m_{s}-l_{i}+l_{i+1}-r+i, \\
& A_{0}^{\vee}(\boldsymbol{l}, k)=-\prod_{s=0}^{r} \frac{\sum_{i=1}^{s} m_{s}-l_{r}+s}{\sum_{i=1}^{s} m_{s}-l_{r}+s+1+l_{r-s}-l_{r-s+1}},
\end{aligned}
$$

where $i=0, \ldots, r-1$.

These numbers satisfy the following property.

Lemma 5.3. We have

$$
A_{i}^{\vee}\left(\boldsymbol{l}-\mathbf{1}_{j}, k-1\right) A_{j}^{\vee}(\boldsymbol{l}, k)=A_{j}^{\vee}\left(\boldsymbol{l}-\mathbf{1}_{i}, k-1\right) A_{i}^{\vee}(\boldsymbol{l}, k) .
$$

In addition

$$
A_{0}^{\vee}((k, \ldots, k), k)=B_{k},
$$

where $B_{k}$ is defined by (2.12). 
The recursion for spaces of polynomials $U(\boldsymbol{m}, \boldsymbol{l}, k)$ translates to the recursion for the first polynomial $u_{0}$.

Proposition 5.4. We have

$$
D_{i}^{\vee}(\boldsymbol{l}, k) u_{0}(\boldsymbol{l}, k)=A_{i}^{\vee}(\boldsymbol{l}, k) u_{0}\left(\boldsymbol{l}-\mathbf{1}_{i}, k-1\right) .
$$

Proof. Similar to the proof of Proposition 4.10.

Let $\boldsymbol{l}$ be a partition such that $\boldsymbol{l}-\mathbf{1}_{i}, \boldsymbol{l}-\mathbf{1}_{j}, \boldsymbol{l}-\mathbf{1}_{s}$ are partitions.

Corollary 5.5. We have

$$
A_{i}^{\vee} A_{j s} u_{0}\left(\boldsymbol{l}-\mathbf{1}_{i}, k-1\right)+A_{j}^{\vee} A_{i s} u_{0}\left(\boldsymbol{l}-\mathbf{1}_{j}, k-1\right)+A_{s}^{\vee} A_{i j} u_{0}\left(\boldsymbol{l}-\mathbf{1}_{s}, k-1\right)=0,
$$

where $A_{t}^{\vee}=A_{t}^{\vee}(\boldsymbol{l}, k), A_{t p}=A_{t p}(\boldsymbol{l}, k)$.

5.3. Explicit formulas. Proposition 5.4 allows us to compute the polynomials $u_{i}$ explicitly.

Write

$$
u_{0}(\boldsymbol{m}, \boldsymbol{l}, k)=\sum_{i=0}^{l_{r}}(-1)^{i} c_{i}(\boldsymbol{m}, \boldsymbol{l}, k) x^{l_{r}-i} .
$$

Proposition 5.6. We have

$$
c_{i}(\boldsymbol{m}, \boldsymbol{l}, k)=\left(\begin{array}{c}
l_{r} \\
i
\end{array}\right) \prod_{j=0}^{i-1} \prod_{s=1}^{r} \frac{\sum_{t=r-s+1}^{r} m_{t}-l_{r}+s+j}{\sum_{t=r-s+1}^{r} m_{t}-l_{r}+s+j+1+l_{r-s}-l_{r-s+1}} .
$$

Proof. We have $c_{0}(\boldsymbol{m}, \boldsymbol{l}, k)=1$. By Proposition 5.4 we have the following relations:

$$
\left(l_{r}-i-e_{r-j}^{\vee}\right) c_{i}(\boldsymbol{m}, \boldsymbol{l}, k)=A_{j}^{\vee}(\boldsymbol{m}, \boldsymbol{l}, k) c_{i}\left(\boldsymbol{m}, \boldsymbol{l}-\mathbf{1}_{j}, k-1\right) .
$$

So, the proposition is obtained by multiplying all the constants.

Note that in fact we computed all $u_{i}$ because of Lemma 5.2

\section{Applications to the Bethe Ansatz}

We consider a special case of the Bethe Ansatz equation. It has at most one solution. If this solution exists, then it is given by zeroes of Jacobi-Piñeiro polynomials and of Wronskians of Jacobi-Piñeiro polynomials. We use properties of Jacobi-Piñeiro polynomials to prove that for generic values of weights, this solution does exist and the corresponding Bethe vector is non-zero. However, we find that in special cases the Bethe equation has no solutions and we obtain a counterexample to the Bethe Ansatz Conjecture for the Gaudin model.

6.1. Generalities of the Bethe Ansatz method. Let $\mathfrak{g}=\mathfrak{n}_{+} \oplus \mathfrak{h} \oplus \mathfrak{n}_{-}$be a simple Lie algebra, (.,.) the Killing form on $\mathfrak{g}, \alpha_{1}, \ldots, \alpha_{r} \in \mathfrak{h}^{*}$ simple roots.

Let $\left(x_{i}\right)_{i \in I}$ be an orthonormal basis in $\mathfrak{g}, \Omega=\sum_{i \in I} x_{i} \otimes x_{i} \in \mathfrak{g} \otimes \mathfrak{g}$ the Casimir element.

For a $\mathfrak{g}$-module $V$ and $\mu \in \mathfrak{h}^{*}$ denote by $V[\mu]$ the weight subspace of $V$ of weight $\mu$ and by $\operatorname{Sing} V[\mu]$ the subspace of singular vectors of weight $\mu$,

$$
\text { Sing } V[\mu]=\left\{v \in V \mid \mathfrak{n}_{+} v=0, h v=\langle\mu, h\rangle v\right\} .
$$


Let $n$ be a positive integer and $\boldsymbol{\Lambda}=\left(\Lambda_{1}, \ldots, \Lambda_{n}\right), \Lambda_{i} \in \mathfrak{h}^{*}$, a set of weights. For a weight $\mu \in \mathfrak{h}^{*}$, let $L_{\mu}$ be the irreducible $\mathfrak{g}$-module with highest weight $\mu$. Denote by $L_{\Lambda}$ the tensor product $L_{\Lambda_{1}} \otimes \cdots \otimes L_{\Lambda_{n}}$.

If $X \in$ End $\left(L_{\Lambda_{i}}\right)$, then we denote by $X^{(i)} \in \operatorname{End}\left(L_{\boldsymbol{\Lambda}}\right)$ the operator $\cdots \otimes$ id $\otimes$ $X \otimes$ id $\otimes \cdots$. If $X=\sum_{k} X_{k} \otimes Y_{k} \in \operatorname{End}\left(L_{\Lambda_{i}} \otimes L_{\Lambda_{j}}\right)$, then we set $X^{(i, j)}=$ $\sum_{k} X_{k}^{(i)} \otimes Y_{k}^{(j)} \in \operatorname{End}\left(L_{\boldsymbol{\Lambda}}\right)$.

Let $\boldsymbol{z}=\left(z_{1}, \ldots, z_{n}\right)$ be a point in $\mathbb{C}^{n}$ with distinct coordinates. Introduce linear operators $K_{1}(\boldsymbol{z}), \ldots, K_{n}(\boldsymbol{z})$ on $L_{\boldsymbol{\Lambda}}$ by the formula

$$
K_{i}(\boldsymbol{z})=\sum_{j, j \neq i} \frac{\Omega^{(i, j)}}{z_{i}-z_{j}}, \quad i=1, \ldots, n .
$$

The operators are called the Gaudin Hamiltonians of the Gaudin model associated with $L_{\boldsymbol{\Lambda}}$. The Hamiltonians commute, $\left[K_{i}(\boldsymbol{z}), K_{j}(\boldsymbol{z})\right]=0$ for all $i, j$.

The problem is to diagonalize simultaneously the Hamiltonians; see $\mathrm{B}, \mathrm{BF}, \mathrm{FFR}$, G, MV1, RV, ScV, V2].

The Hamiltonians commute with the $\mathfrak{g}$-action on $L_{\boldsymbol{\Lambda}}$; thus it is enough to diagonalize the Hamiltonians on the subspaces of singular vectors, $\operatorname{Sing} L_{\boldsymbol{\Lambda}}[\mu] \subset L_{\boldsymbol{\Lambda}}$.

The eigenvectors of the Gaudin Hamiltonians are constructed by the Bethe Ansatz method.

Fix $\Lambda_{\infty}=\sum_{s=1}^{n} \Lambda_{s}-\sum_{i=1}^{r} l_{i} \alpha_{i}$ where $l_{1}, \ldots, l_{r}$ are non-negative integers. Set

$$
\boldsymbol{t}=\left(t_{1}^{(1)}, \ldots, t_{l_{1}}^{(1)}, t_{1}^{(2)}, \ldots, t_{l_{2}}^{(2)}, \ldots, t_{1}^{(r)}, \ldots, t_{l_{r}}^{(r)}\right) .
$$

One defines a suitable rational function $w(\boldsymbol{t}, \boldsymbol{z})$ with values in $L_{\boldsymbol{\Lambda}}\left[\Lambda_{\infty}\right]$ as in $[\mathrm{RV}]$; cf. MV2, RSV. The function is called canonical. The canonical function is symmetric with respect to the group $\Sigma_{l}=\Sigma_{l_{1}} \times \cdots \times \Sigma_{l_{r}}$ of permutations of coordinates $t_{j}^{(i)}$ with the same upper index. One considers the system of equations

$$
-\sum_{s=1}^{n} \frac{\left(\Lambda_{s}, \alpha_{i}\right)}{t_{j}^{(i)}-z_{s}}+\sum_{s, s \neq i} \sum_{k=1}^{l_{s}} \frac{\left(\alpha_{s}, \alpha_{i}\right)}{t_{j}^{(i)}-t_{k}^{(s)}}+\sum_{s, s \neq j} \frac{\left(\alpha_{i}, \alpha_{i}\right)}{t_{j}^{(i)}-t_{s}^{(i)}}=0,
$$

where $i=1, \ldots, r$ and $j=1, \ldots, l_{i}$. The system is symmetric with respect to $\Sigma_{l}$. One shows that if $\boldsymbol{t}^{0}$ is a solution to (6.1), then $w\left(\boldsymbol{t}^{0}, \boldsymbol{z}\right)$ belongs to Sing $L_{\boldsymbol{\Lambda}}\left[\Lambda_{\infty}\right]$ and $w\left(\boldsymbol{t}^{0}, \boldsymbol{z}\right)$ is an eigenvector of the Hamiltonians $K_{1}(\boldsymbol{z}), \ldots, K_{n}(\boldsymbol{z})$; see [RV].

This method of finding eigenvectors is called the Bethe Ansatz method. System (6.1) is called the Bethe Ansatz equation, and the vector $w\left(\boldsymbol{t}^{0}, \boldsymbol{z}\right)$ is called a Bethe vector.

The standard form of the Bethe Ansatz Conjecture says that if $\Lambda_{1}, \ldots, \Lambda_{n}, \Lambda_{\infty}$ are integral dominant, and $z_{1}, \ldots, z_{n}$ are generic, then the Bethe vectors form a basis in Sing $L_{\boldsymbol{\Lambda}}\left[\Lambda_{\infty}\right]$. In particular, the conjecture implies that the number of $\Sigma_{l}$-orbits of solutions to (6.1) is not less than the dimension of $\operatorname{Sing} L_{\Lambda}\left[\Lambda_{\infty}\right]$.

6.2. Specialized Bethe Ansatz equation. Fix a natural number $r$ and a sequence of non-negative integers $\boldsymbol{m}$. Choose a sequence of non-negative integers $\boldsymbol{l}$ and a non-negative integer $k$. 
Consider the following system of algebraic equations for variables $\boldsymbol{t}=\left(t_{i}^{(j)}\right)$, $i=1, \ldots, l_{j}, j=1, \ldots, r$ :

$$
\begin{aligned}
& \frac{m_{1}}{t_{i}^{(1)}}+\frac{k}{t_{i}^{(1)}-1}-\sum_{s, s \neq i} \frac{2}{t_{i}^{(1)}-t_{s}^{(1)}}+\sum_{s} \frac{1}{t_{i}^{(1)}-t_{s}^{(2)}}=0 \quad\left(i=1, \ldots, l_{1}\right), \\
& \frac{m_{j}}{t_{i}^{(j)}}-\sum_{s, s \neq i} \frac{2}{t_{i}^{(j)}-t_{s}^{(j)}}+\sum_{s} \frac{1}{t_{i}^{(j)}-t_{s}^{(j+1)}}+\sum_{s} \frac{1}{t_{i}^{(j)}-t_{s}^{(j-1)}}=0 \quad\left(i=1, \ldots, l_{j}\right), \\
& \frac{m_{r}}{t_{i}^{(r)}}-\sum_{s, s \neq i} \frac{2}{t_{i}^{(r)}-t_{s}^{(r)}}+\sum_{s} \frac{1}{t_{i}^{(r)}-t_{s}^{(r-1)}}=0 \quad\left(i=1, \ldots, l_{r}\right),
\end{aligned}
$$

where $j=2, \ldots, r-1$.

System (6.2) is the Bethe Ansatz equation (6.1) for the Gaudin model associated to the Lie algebra $s l_{r+1}$, points $z_{1}=0, z_{2}=1$, and weights $\Lambda_{1}, \Lambda_{2}$, such that the scalar products with simple roots are given by (2.1). The Bethe Ansatz equation with two arbitrary points $z_{1}$ and $z_{2}$ is related to our choice of $z_{1}=0$ and $z_{1}=1$ by a simple rescaling of the variables $t$.

System (6.2) is invariant with respect to the group $\Sigma_{l}=\Sigma_{l_{1}} \times \cdots \times \Sigma_{l_{r}}$ of permutations of variables with the same upper index. Thus the group acts on solutions of the system. This action is free, and we do not distinguish between solutions in the same orbit.

Assume that the weight

$$
\Lambda_{\infty}=\Lambda_{1}+\Lambda_{2}-\sum_{s} l_{s} \alpha_{s}
$$

is dominant. Then it is known, see MV1, that the number of orbits of solutions of equation (6.2) is finite and it is bounded from above by the dimension of the space of singular vectors of weight $\Lambda_{\infty}$ in the tensor product $L_{\Lambda_{1}} \otimes L_{\Lambda_{2}}$. Using Lemma 2.1. we obtain the following lemma.

Lemma 6.1. If $\Lambda_{\infty}$ is dominant, then system (6.2) has no solutions unless $\boldsymbol{m}, \boldsymbol{l}, \boldsymbol{k}$ are consistent. In that case the system has at most one orbit of solutions.

For $\boldsymbol{t}=\left(t_{i}^{j}\right)$, we define an $r$-tuple of polynomials $\boldsymbol{y}=\left(y_{1}, \ldots, y_{r}\right)$,

$$
y_{i}=\prod_{s=1}^{l_{i}}\left(x-t_{s}^{(i)}\right) .
$$

We are interested only in zeroes of $y_{i}$, and we consider each polynomial $y_{i}$ up to multiplication by a non-zero complex number. We say that $\boldsymbol{y}$ represents $\boldsymbol{t}$. Note that $\operatorname{deg} y_{i}=l_{i}$.

We call an $r$-tuple of polynomials $\left(y_{1}, \ldots, y_{r}\right)$ generic if all $y_{i}$ have no multiple roots, no common roots with $y_{i \pm 1}$ and no common roots with $T_{i}$, where $T_{i}$ are given by (2.11). If $\boldsymbol{y}$ represents a solution $\boldsymbol{t}$ of the Bethe Ansatz equation, then $\boldsymbol{y}$ is generic.

6.3. Connection between Bethe Ansatz and spaces of polynomials. We fix a choice of consistent parameters $\boldsymbol{m}, \boldsymbol{l}, k$. Let $U=U(\boldsymbol{m}, \boldsymbol{l}, k)$ and $V=V(\boldsymbol{m}, \boldsymbol{l}, k)$ be the corresponding spaces of polynomials; see Lemmas $2.3,2.2$. 
Let $f_{1}, \ldots, f_{r+1}$ and $g_{1}, \ldots, g_{r+1}$ be bases of $V$ and $U$ as in Lemmas 4.4 and 5.1 Then it is shown in MV1], cf. also Lemma 2.5, that polynomials $W^{\dagger}\left(f_{1}, \ldots, f_{i}\right)$ and $W^{\dagger}\left(g_{1}, \ldots, g_{r+1-i}\right)$ are equal up to multiplication by a non-zero constant. Let $y_{i}$ be the monic constant multiple of $W^{\dagger}\left(f_{1}, \ldots, f_{i}\right)$. We have

$$
y_{i}=c W^{\dagger}\left(f_{1}, \ldots, f_{i}\right) \quad(i=1, \ldots, r),
$$

where $c$ is a non-zero constant depending on $i$ and $f_{1}, \ldots, f_{i}$.

For example in the case of $l_{1}=\cdots=l_{r}=0$, we have $y_{1}=\cdots=y_{r}=1$.

Lemma 6.2. The Bethe Ansatz equation (6.2) for consistent $\boldsymbol{m}, \boldsymbol{l}, k$ has a solution $\boldsymbol{t}$ if and only if the $r$-tuple $\boldsymbol{y}=\left(y_{1}, \ldots, y_{r}\right)$ is generic. Moreover, in such a case, $\boldsymbol{y}$ represents $\boldsymbol{t}$.

Proof. If the Bethe Ansatz equation (6.2), associated with consistent parameters $\boldsymbol{m}, \boldsymbol{l}, k$, has a solution $\boldsymbol{t}$, then the solution $\boldsymbol{t}$ generates "a population of solutions"; see Section 3.4 in [MV1]. That population of solutions has its "fundamental space" $V_{\boldsymbol{t}}$, which is an $(r+1)$-dimensional vector space of polynomials with exactly two singular points at 0 and 1 and exponents given by (2.6); see Section 5.3 in [MV1. Thus $V_{\boldsymbol{t}}=V(\boldsymbol{m}, \boldsymbol{l}, k)$ by Lemma 2.2. Given the fundamental space $V_{\boldsymbol{t}}$, define the tuple $\boldsymbol{y}$ by formula (6.3). Then $\boldsymbol{y}$ represents the solution $\boldsymbol{t}$; see Theorem 5.12 in MV1. Since $\boldsymbol{t}$ is a solution of the Bethe Ansatz equation, the tuple $\boldsymbol{y}$ is generic in the sense of Section 6.2. This proves the lemma.

6.4. Properties of polynomials $y_{1}, \ldots, y_{r}$. Let $\boldsymbol{m}, \boldsymbol{l}, k$ be consistent parameters. We have the corresponding monic polynomials $y_{i}(\boldsymbol{m}, \boldsymbol{l}, k)$; see (6.3) and Lemma 6.2 .

Proposition 6.3. For all consistent parameters $\boldsymbol{m}, \boldsymbol{l}, k$ we have

$$
y_{1}(\boldsymbol{m}, \boldsymbol{l}, k)(1) \neq 0, \quad y_{i}(\boldsymbol{m}, \boldsymbol{l}, k)(0) \neq 0 \quad(i=1, \ldots, r) .
$$

Proof. The proposition holds if and only if $F_{r+1-s}(0) \cap F_{s}(\infty)=0$ and $F_{r}(1) \cap$ $F_{1}(\infty)=0$ in $V(\boldsymbol{m}, \boldsymbol{l}, k)$. The proposition is obviously true if $(\boldsymbol{l}, k)=(\mathbf{0}, 0)$. Now the proposition follows for all consistent parameters $\boldsymbol{m}, \boldsymbol{l}, k$ because there exists a canonical map $V(\boldsymbol{m}, \mathbf{0}, 0) \rightarrow V(\boldsymbol{m}, \boldsymbol{l}, k)$.

Note that Proposition 6.3 shows that the polynomials $y_{i}$ and $T_{i}$ are relatively prime.

Lemma 6.4. The polynomials $y_{i}(\boldsymbol{m}, \boldsymbol{l}, k), i=1, \ldots, r$, are polynomials of degree $l_{i}$ whose coefficients are rational functions of $\boldsymbol{m}, k$ with coefficients in $\mathbb{Q}$.

Proof. From Theorem 3.3 it is clear that we have a basis in $V(\boldsymbol{m}, \boldsymbol{l}, k)$ which is compatible with the flag at infinity and whose coefficients are rational functions of $\boldsymbol{m}, k$. Clearly, we still have rational coefficients after the operation of taking the divided Wronskian.

Lemma 6.4 allows us to consider polynomials $y_{i}(\boldsymbol{m}, \boldsymbol{l}, k)$ for all generic complex parameters $\boldsymbol{m}, k$.

The next two lemmas describe relations between polynomials corresponding to different $r$.

Let $\overline{\boldsymbol{m}}=\left(m_{2}, \ldots, m_{r}\right), \overline{\boldsymbol{l}}=\left(l_{2}, \ldots, l_{r}\right)$. 
Lemma 6.5. We have

$$
\begin{aligned}
\lim _{m_{1} \rightarrow \infty} y_{1}(\boldsymbol{m}, \boldsymbol{l}, k) & =(x-1)^{l_{1}}, \\
\lim _{m_{1} \rightarrow \infty} y_{i}(\boldsymbol{m}, \boldsymbol{l}, k) & =y_{i-1}\left(\overline{\boldsymbol{m}}, \overline{\boldsymbol{l}}, l_{1}\right),
\end{aligned}
$$

where $i=2, \ldots, r$.

Proof. Recall the basis of $V(\boldsymbol{m}, \boldsymbol{l}, k)$ described in Lemma 4.4. From (4.2) we see that the $v_{i}$ have finite limits when $m_{1}$ tends to infinity. Denote these limits by $\bar{v}_{i}$. From Proposition 4.10 we conclude that $\bar{v}_{0}=(x-1)^{l_{1}}$. Consider the space

$$
\left\{\bar{v}_{1}, x^{m_{2}+1} \bar{v}_{2}, \ldots, x^{\sum_{i=2}^{r} m_{i}+i-1} \bar{v}_{r}\right\} .
$$

This space has the same exponents as $V\left(\overline{\boldsymbol{m}}, \overline{\boldsymbol{l}}, l_{1}\right)$. Indeed, it is clear for exponents at infinity. The exponents in the finite points can only increase in the limit. It does not happen because of relation (2.5).

Therefore this space coincides with $V\left(\overline{\boldsymbol{m}}, \overline{\boldsymbol{l}}, l_{1}\right)$. The lemma follows.

Let $\tilde{\boldsymbol{m}}=\left(m_{1}, \ldots, m_{r}, n\right)$, where $n$ is any complex number, $\tilde{\boldsymbol{l}}=\left(l_{1}, \ldots, l_{r}, 0\right)$.

Lemma 6.6. We have

$$
\begin{aligned}
& y_{i}(\tilde{\boldsymbol{m}}, \tilde{\boldsymbol{l}}, k)=y_{i}\left(\boldsymbol{m}, \boldsymbol{l}, l_{1}\right), \\
& y_{r+1}(\tilde{\boldsymbol{m}}, \tilde{\boldsymbol{l}}, k)=1,
\end{aligned}
$$

where $i=1, \ldots, r$.

Proof. The lemma follows from Lemma 3.13 .

Finally we describe the properties of zeros of $y_{i}$ in a certain asymptotic zone.

Lemma 6.7. Fix a partition $\boldsymbol{l}$ and a negative real number $k$. Let $\boldsymbol{m}$ be a sequence of real numbers such that

$$
m_{1} \ll m_{2} \ll \cdots \ll m_{r} \ll 0 .
$$

Then the corresponding $r$-tuple $\boldsymbol{y}$ is generic. Moreover all $y_{i}$ have real distinct roots $t_{i}^{(j)}$ such that $0<t_{i}^{(j)}<1$ and $t_{i_{1}}^{\left(j_{1}\right)}>t_{i_{2}}^{\left(j_{2}\right)}$ if $j_{1}<j_{2}$.

Proof. If all $m_{i}<0$, then all roots of $y_{1}(\boldsymbol{m}, \boldsymbol{l}, k)$ are distinct, real and located in $(0,1)$. This is a general fact about roots of multiple orthogonal polynomials; see IN].

Let $m_{1} \ll 0$, and let all other $m_{i}$ be real. Then the roots of $y_{2}$ tend to the roots of another multiple orthogonal polynomial by Lemma 6.5. Therefore the roots of $y_{2}$ are also real and distinct. Moreover, they are smaller than the roots of $y_{1}$, which all are close to one.

Let $m_{1} \ll m_{2} \ll 0$, and let all other $m_{i}$ be real. Then the roots of $y_{3}$ also tend to the roots of a multiple orthogonal polynomial by Lemma 6.5. Therefore the roots of $y_{3}$ are also real and distinct. Moreover, they are smaller than the roots of $y_{2}$, which are close to one in this zone.

The lemma follows. 
6.5. Bethe vectors. Let $l_{1}, \ldots, l_{r}, k$ be non-negative integers such that $k \geq l_{1} \geq$ $\cdots \geq l_{r}$. Let $m_{i}$ be real numbers.

We list properties of the canonical function used for the construction of the Bethe vectors $w(\boldsymbol{t})=w(\boldsymbol{t}, 0,1) \in L_{\boldsymbol{\Lambda}}\left[\Lambda_{\infty}\right]$; see [RSV].

(i) We have $L_{\boldsymbol{\Lambda}}\left[\Lambda_{\infty}\right]=\bigoplus_{\mu_{1}+\mu_{2}=\Lambda_{\infty}} L_{\Lambda_{1}}\left[\mu_{1}\right] \otimes L_{\lambda_{2}}\left[\mu_{2}\right]$. For every such $\mu_{1}, \mu_{2}$, there exists a rational function $w_{\mu_{1}, \mu_{2}}(\boldsymbol{t}) \in L_{\Lambda_{1}}\left[\mu_{1}\right] \otimes L_{\lambda_{2}}\left[\mu_{2}\right]$ such that $w(\boldsymbol{t})=\sum_{\mu_{1}+\mu_{2}=\Lambda_{\infty}} w_{\mu_{1}, \mu_{2}}(\boldsymbol{t})$.

(ii) Let $F_{1}, \ldots, F_{r} \in \mathfrak{n}_{-}$be the standard generators. Let $U\left(\mathfrak{n}_{-}\right)$be the complex universal enveloping algebra of $\mathfrak{n}_{-}$. Let $U^{\mathbb{R}}\left(\mathfrak{n}_{-}\right) \subset U\left(\mathfrak{n}_{-}\right)$be its real part (consisting of polynomials in $F_{1}, \ldots, F_{r}$ with real coefficients). For $s=1,2$, let $v_{s} \in L_{\Lambda_{s}}$ be a highest weight vector. Let $L_{\Lambda_{s}}^{\mathbb{R}}$ be the real part of $L_{\Lambda_{s}}$; i.e., $L_{\Lambda_{s}}^{\mathbb{R}}$ is the image of $v_{s}$ under the action of $U^{\mathbb{R}}\left(\mathfrak{n}_{-}\right)$. For all $\mu_{1}, \mu_{2}$, we have $w_{\mu_{1}, \mu_{2}}(\boldsymbol{t}) \in L_{\Lambda_{1}}^{\mathbb{R}}\left[\mu_{1}\right] \otimes L_{\lambda_{2}}^{\mathbb{R}}\left[\mu_{2}\right]$ if all coordinates $t_{j}^{(i)}$ of the vector $\boldsymbol{t}$ are real.

(iii) Let $\boldsymbol{\Sigma}_{l}=\prod_{i} \Sigma_{l_{i}}$ be the product of symmetric groups. Let $\nu$ be a function of $\boldsymbol{t}$. We define the action of $\pi \in \boldsymbol{\Sigma}_{\boldsymbol{l}}$ on $\nu$ by permuting the $t_{j}^{(i)}$,s with the same upper index. We define the symmetrizer operator $\operatorname{Sym} \nu=\sum_{\pi \in \Sigma_{l}} \pi \cdot \nu$. We have the following formula:

$$
\begin{aligned}
& w_{\Lambda_{1}, \Lambda_{2}-\sum_{i=1}^{r} l_{i} \alpha_{i}}(\boldsymbol{t}) \\
& \quad=\nu(\boldsymbol{t}) v_{1} \otimes F_{1}^{l_{1}-l_{2}}\left[F_{2}, F_{1}\right]^{l_{2}-l_{3}} \ldots\left[F_{r},\left[F_{r-1},\left[\ldots,\left[F_{2}, F_{1}\right] \ldots\right]\right]\right]^{l_{r}} v_{2},
\end{aligned}
$$

where

$$
\begin{aligned}
& \nu(\boldsymbol{t})=\frac{1}{\left(l_{1}-l_{2}\right) ! \ldots\left(l_{r-1}-l_{r}\right) ! l_{r} !} \\
& \times \operatorname{Sym}\left[\prod_{i=1}^{l_{1}-l_{2}} \frac{1}{\left(t_{i}^{(1)}-1\right)} \prod_{i=1}^{l_{2}-l_{3}} \frac{1}{\left(t_{i+l_{1}-l_{2}}^{(1)}-1\right)\left(t_{i}^{(2)}-t_{i+l_{1}-l_{2}}^{(1)}\right)} \ldots\right. \\
& \left.\prod_{i=1}^{l_{r}} \frac{1}{\left(t_{i+l_{1}-l_{r}}^{(1)}-1\right)\left(t_{i+l_{2}-l_{r}}^{(2)}-t_{i+l_{1}-l_{r}}^{(1)}\right)\left(t_{i+l_{3}-l_{r}}^{(3)}-t_{i+l_{2}-l_{r}}^{(2)}\right) \ldots\left(t_{i}^{(r)}-t_{i+l_{r-1}-l_{r}}^{(r-1)}\right)}\right] .
\end{aligned}
$$

Theorem 6.8. Fix a partition $\boldsymbol{l}$. Then for generic complex values of $k, m_{1}, \ldots, m_{r}$ we have:

- system (6.2) has a unique orbit of solutions,

- the multiplicity of this solution is equal to one,

- the corresponding Bethe vector is non-zero.

Proof. Consider the $r$-tuple $\boldsymbol{y}(\boldsymbol{m}, \boldsymbol{l}, k)=\left(y_{1}(\boldsymbol{m}, \boldsymbol{l}, k), \ldots, y_{r}(\boldsymbol{m}, \boldsymbol{l}, k)\right)$ associated with parameters $\boldsymbol{m}, \boldsymbol{l}, k$. By Lemma 6.7 the $r$-tuple is generic for generic complex values of the parameters $\boldsymbol{m}, k$. Hence for almost all consistent values of the parameters $\boldsymbol{m}, \boldsymbol{l}, k$ with fixed $\boldsymbol{l}$, the $r$-tuple $\boldsymbol{y}(\boldsymbol{m}, \boldsymbol{l}, k)$ is generic. The exceptions lie in a proper algebraic subset in the space of all $\boldsymbol{m}, k$.

Hence by Lemma 6.1 for almost all consistent $\boldsymbol{m}, \boldsymbol{l}, k$ with fixed $\boldsymbol{l}$ the Bethe Ansatz equation has exactly one orbit of solutions. Equisingularity theorems in V1 imply that the Bethe Ansatz equation has exactly one orbit for almost all complex values of the parameters $\boldsymbol{m}, k$ with fixed $\boldsymbol{l}$.

Let us prove that the solutions of that only orbit have multiplicity one. Indeed if $\boldsymbol{m}, \boldsymbol{l}, k$ are as in Lemma 6.7, then using the properties of the weight function 
described above, we obtain that the corresponding Bethe vector $w(t)$ is non-zero and belongs to the real part of $L_{\boldsymbol{\Lambda}}$. Then the multiplicity of solutions of the only orbit is equal to one by Corollary 7.2 in MV2. Hence the multiplicity of solutions of the only orbit is equal to one for almost all values of $\boldsymbol{m}, k$. On the other hand, if the multiplicity is one, then the corresponding Bethe vector is non-zero; see MV2.

6.6. A counterexample to the Bethe Ansatz Conjecture. According to Theorem 6.8, for generic values of the parameters, there is a unique solution of the Bethe Ansatz equation (6.2) and a unique non-zero Bethe vector. In particular, for generic values of $\boldsymbol{m}, k$ the Bethe Ansatz Conjecture holds. In this section we give an example of parameters when this is not true.

We consider the case of $r=2, l_{1}=2, l_{2}=1$. Then the polynomials $y_{1}$ and $y_{2}$ are computed to be

and

$$
y_{2}=x-\frac{m_{2}\left(m_{1}+m_{2}+1\right)}{\left(m_{2}+2\right)\left(k+m_{1}+m_{2}\right)}
$$

$y_{1}=(x-1)^{2}+\frac{(k-1)\left(2 k+m_{2}+2 m_{2}\right)}{\left(m_{1}+k-1\right)\left(m_{1}+m_{2}+k\right)}(x-1)+\frac{k(k-1)}{\left(m_{2}+m_{1}+k\right)\left(m_{1}+k-1\right)}$.

Lemma 6.9. Suppose $\left(2 m_{1}+m_{2}\right)^{2}+k\left(4 m_{1}-m_{2}^{2}\right)=0$. Then we have $y_{1}=y_{2}^{2}$. In particular the pair $\left(y_{1}, y_{2}\right)$ is not generic.

Let

$$
r=2, \quad k=49, \quad m_{1}=2, \quad m_{2}=3, \quad l_{1}=2, \quad l_{2}=1 .
$$

Define $s l_{3}$ weights $\Lambda_{1}, \Lambda_{2}$ by the conditions $\left(\Lambda_{1}, \alpha_{i}\right)=m_{i},\left(\Lambda_{2}, \alpha_{i}\right)=k \delta_{1 i}$. Let $\Lambda_{\infty}=\Lambda_{1}+\Lambda_{2}-l_{1} \alpha_{1}-l_{2} \alpha_{2}$. Let $L_{\Lambda}$ be the irreducible $s l_{3}$ module of highest weight $\Lambda$.

Proposition 6.10. The multiplicity of $L_{\Lambda_{\infty}}$ in $L_{\Lambda_{1}} \otimes L_{\Lambda_{2}}$ is 1 . The corresponding Bethe equation (6.2) has no solutions.

Proof. The parameters $\boldsymbol{m}, \boldsymbol{l}, k$ are consistent; therefore the multiplicity of $L_{\Lambda_{\infty}}$ in $L_{\Lambda_{1}} \otimes L_{\Lambda_{2}}$ is one by Lemma 2.1 The pair $y_{1}(\boldsymbol{m}, \boldsymbol{l}, k), y_{2}(\boldsymbol{m}, \boldsymbol{l}, k)$ is not generic by Lemma 6.9 therefore system (6.2) has no solutions.

Proposition 6.10 provides a counterexample for the standard version of the Bethe Ansatz Conjecture for the Gaudin model.

Remark. Here is another counterexample to the standard version of the Bethe Ansatz conjecture.

Consider the tensor product of two adjoint $s l_{3}$ representations. Then the multiplicity of the trivial module in this tensor product is 1 . One can explicitly check that the corresponding 3 -dimensional vector space $V$ is

$$
V=\operatorname{span}\left\{(2 x-1)^{2},(x-1)^{4}, x^{4}\right\} .
$$

The space $V$ is the unique 3-dimensional space of polynomials spanned by polynomials of degrees 2,3 and 4 which has two finite singular points 0,1 such that the exponents at both singular points are $0,2,4$.

Then the corresponding pair $\left(y_{1}, y_{2}\right)=\left((x-1 / 2)^{2},(x-1 / 2)^{2}\right)$ is not generic and by the same argument as in Proposition 6.10, the corresponding Bethe Ansatz equation has no solutions. 


\section{ApPENDix}

7.1. A scalar product. We compute the scalar product of $y_{1}$ and $y_{r}$ with respect to the measure defined by integration with the Jacobi-type weight.

Let

$$
\omega_{m, k}=(x-1)^{-k-1} x^{-m-1} .
$$

Set

$$
|\boldsymbol{m}|=\sum_{i=1}^{r} m_{i}+r-1 .
$$

Then we have $\omega_{|\boldsymbol{m}|, k}=w$, where $\omega$ is given by (4.1).

Assume that $m$ and $k$ are negative real numbers. Define a scalar product of functions $f(x)$ and $g(x)$ by the formula

$$
(f(x), g(x))_{m, k}=\int_{0}^{1} f(x) g(x) \omega_{m, k}(x) d x .
$$

The Jacobi-Piñeiro polynomial $P(\boldsymbol{m}, \boldsymbol{l}, k)$ is the unique monic polynomial of degree $l_{1}$ which has the property

$$
\left(P, x^{s}\right)_{|\boldsymbol{m}|, k}=0
$$

for all $s$ of the form

$$
s=\sum_{j=r-i+1}^{r} m_{j}+t, \quad i \in\{0,1, \ldots, r-1\}, t-i \in\left\{0,1, \ldots, l_{r-i}-l_{r-i+1}-1\right\},
$$

where $k$ is a negative number and the $m_{j}$ are such that $\sum_{j=0}^{i} m_{j}+i-1<0$ for $i=1, \ldots, r$.

Operators $D_{i}$ and $D_{i}^{\vee}$ are adjoint in the following sense:

Lemma 7.1. We have

$$
\left(D_{i}(\boldsymbol{m}, \boldsymbol{l}, k) f, g\right)_{|\boldsymbol{m}|+1, k+1}=-\left(f, D_{i}^{\vee}\left(\boldsymbol{m}, \boldsymbol{l}+\mathbf{1}_{i}, k+1\right) g\right)_{|\boldsymbol{m}|+1, k} .
$$

Proof. The lemma is checked by integration by parts.

Using Lemma 7.1 and Propositions 4.10 and 5.4 , the following scalar product is easy to compute explicitly:

Lemma 7.2. We have

$$
\begin{aligned}
&\left(y_{1}(\boldsymbol{m}, \boldsymbol{l}, k), y_{r}(\boldsymbol{m}, \boldsymbol{l}, k)\right)_{|\boldsymbol{m}|+1, k} \\
&=(1,1)_{|\boldsymbol{m}|+1, k-l_{1}} \times \prod_{i=0}^{r-1}\left(\prod_{j=0}^{l_{r}-1} \frac{\sum_{t=r-j}^{r} m_{t}+i-j}{\sum_{t=r-j}^{r} m_{t}+i-j+1+l_{r-i-1}-l_{r-i}}\right. \\
&\left.\times \prod_{s=0}^{l_{i}-l_{i+1}-1} \frac{\sum_{t=i+1}^{r} m_{t}+r+1-i+s}{\sum_{t=1}^{i} m_{t}+i-s+k-l_{1}}\right) .
\end{aligned}
$$


7.2. Affine Weyl group. In this section we note that operators $D_{i}$ together with the Weyl group generate an extension of the affine Weyl group.

We call parameters $\boldsymbol{m}, \boldsymbol{l}, k$ admissible if the polynomials $v_{i}(\boldsymbol{m}, \boldsymbol{l}, k), i=0, \ldots, r$, are well defined. For admissible parameters $\boldsymbol{m}, \boldsymbol{l}, \boldsymbol{k}$, we define the corresponding space $V(\boldsymbol{m}, \boldsymbol{l}, k)$ as follows. The space $V(\boldsymbol{m}, \boldsymbol{l}, k)$ is the space of functions with the explicit linear basis $\left\{v_{0}(\boldsymbol{m}, \boldsymbol{l}, k), v_{1}(\boldsymbol{m}, \boldsymbol{l}, k) x^{m_{1}+1}, \ldots, v_{r}(\boldsymbol{m}, \boldsymbol{l}, k) x^{\sum_{j=1}^{r} m_{j}+r}\right\}$, where $v_{i}(\boldsymbol{m}, \boldsymbol{l}, k)$ are given by (4.2).

Let $V$ be a disjoint direct sum of spaces $V(\boldsymbol{m}, \boldsymbol{l}, k)$,

$$
V=\bigoplus_{\boldsymbol{m}, \boldsymbol{l}, k} V(\boldsymbol{m}, \boldsymbol{l}, k)
$$

where the sum is taken over all sequences $\boldsymbol{m}$ of $r$ complex numbers, all sequences $\boldsymbol{l}$ of non-negative integers and all non-negative integers $k$ such that $\boldsymbol{m}, \boldsymbol{l}, k$ are admissible and $k \geq l_{1} \geq \cdots \geq l_{r}$.

The Weyl group $\mathcal{S}_{r+1}$ acts on $V$ by acting on parameters. Namely, we define the action of the Weyl group on $V$ by the following rule:

$$
\begin{aligned}
s_{i}: V(\boldsymbol{m}, \boldsymbol{l}, k) & \rightarrow V\left(s_{i} \cdot \boldsymbol{m},\left(s_{i}\right)_{k} \boldsymbol{l}, k\right), \\
v_{j}(\boldsymbol{m}, \boldsymbol{l}, k) x^{\sum_{t=1}^{j} m_{t}+j} & \mapsto v_{s_{i}(j)}\left(s_{i} \cdot \boldsymbol{m},\left(s_{i}\right)_{k} \boldsymbol{l}, k\right) x^{\sum_{t=1}^{s_{i}(j)} m_{t}+s_{i}(j)},
\end{aligned}
$$

where $i=1, \ldots, r$ and $j=0, \ldots, r$.

Introduce linear operators $D_{i}, i=0, \ldots, r$, acting on $V$. We set

$$
D_{i}: V(\boldsymbol{m}, \boldsymbol{l}, k) \rightarrow V\left(\boldsymbol{m}, \boldsymbol{l}+\mathbf{1}_{i}, k+1\right)
$$

so that the restriction of operator $D_{i}$ to $V(\boldsymbol{m}, \boldsymbol{l}, k)$ is equal to $D_{i}(\boldsymbol{m}, \boldsymbol{l}, k)$.

We have the following commutation relations.

Lemma 7.3. We have

$$
D_{i} D_{j}=D_{j} D_{i}, \quad s_{i} D_{j}=D_{s_{i}(j)} s_{i} .
$$

Consider the semigroup $G$ generated by $s_{i}, i=1, \ldots, r$, and $D_{i}, i=0, \ldots, r$, subject to the Weyl group relations $s_{i}^{2}=i d, s_{i} s_{i+1} s_{i}=s_{i+1} s_{i} s_{i+1}, s_{i} s_{j}=s_{j} s_{i}$ $(|i-j|>1)$ and relations (7.1).

Note that the element $D_{0} \ldots D_{r}$ is in the center of $G$. The factor of $G$ by the sub-semigroup generated by this element is isomorphic to the affine Weyl group of $s l_{r+1} ;$ cf. [K], Proposition 6.5.

\section{REFERENCES}

[ABV] A. I. Aptekarev, A. Branquinho, W. Van Assche, Multiple orthogonal polynomials for classical weights, Transactions of the AMS, 355, no. 10, 3887-3914 MR1990569|(2004g:33014)

[B] H. Babujian, Off-shell Bethe ansatz equations and $N$-point correlators in the $\mathrm{SU}(2)$ WZNW theory, J. Phys. A 26 (1993), no. 23, 6981-6990 MR.1253889 (95a:82028)

[BF] H. Babujian and R. Flume, Off-shell Bethe ansatz equation for Gaudin magnets and solutions of Knizhnik-Zamolodchikov equations, Modern Phys. Lett. A 9 (1994), n. 22, 2029-2039 MR:1290286 (95h:82007)

[EH] D. Eisenbud, J. Harris, Limit Linear Series: Basic Theory, Inventiones Mathematicae, 85, 337-371 MR846932 (87k:14024)

[FFR] B. Feigin, E. Frenkel, and N. Reshetikhin, Gaudin model, Bethe Ansatz and Critical Level, Commun. Math. Phys. 166 (1994), 29-62 MR1309540 (96e:82012)

[G] M. Gaudin, Diagonalisation d'une classe d'Hamiltoniens de spin, J. Physique 37 (1976), no. 10, 1089-1098 MR0421442 (54:9446)

[IN] A. Iserles, S.P. Norsett, On the theory of bi-orthogonal polynomials, Transactions of AMS, 306 (1988), 455-474 MR 933301 (89c:42027) 
[K] V. Kac, Infinite-dimensional Lie algebras, Cambridge University Press, 1990 MR 1104219 (92k:17038)

[MV1] E. Mukhin, A. Varchenko, Critical points of master functions and flag varieties, Communications in Contemporary Mathematics (2004), vol. 6, no. 1, 111-163 MR2048778 (2005b:17052)

[MV2] E. Mukhin, A. Varchenko, Norm of a Bethe Vector and the Hessian of the Master Function, Compos. Math. 141 (2005), no. 4, 1012-1028 MR2148192 (2006d:82022)

[MV3] E. Mukhin, A. Varchenko, Solutions to the XXX type Bethe Ansatz equations and flag varieties, Centr. Eur. J. Math, vol. 1, no.2 (2003), 238-271 MR.1993451 (2004k:82026)

[P] L. R. Piñeiro, On simultaneous Padé approximants for a collection of Markov functions, Vestnik Mosk. Univ. Ser., I, no. 2 (1987), 52-55 (in Russian); translated in Moscow Univ. Math. Bull. 42, no. 2 (1987), 52-55

[PV] K. Postelmans, W. Van Assche, Multiple little q-Jacobi polynomials, math.CA/0403532, $1-15$

[RSV] R. Rimanyi, L. Stevens, and A. Varchenko, Combinatorics of rational functions and Poincaré-Birkhoff-Witt expansions of the canonical $U\left(\mathfrak{n}_{-}\right)$-valued differential form, math.CO/0407101, 1-14

[RV] N. Reshetikhin, A. Varchenko, Quasiclassical asymptotics of solutions to the KZ equations, Geometry, topology \& physics, Conf. Proc. Lecture Notes Geom. Topology, VI, Internat. Press, Cambridge, MA (1995), 293-322 MR1358621(96j:32025)

[Sc] I. Scherbak, Intersections of Schubert varieties and highest weight vectors in tensor products of $s_{N+1}$ representations, math.RT/0409329, 1-23

$[\mathrm{ScV}]$ I. Scherbak, A. Varchenko, Critical point of functions, sl $_{2}$ representations and Fuchsian differential equations with only univalued solutions, Dedicated to Vladimir I. Arnold on the occasion of his 65th birthday. Mosc. Math. J. 3 (2003), no. 2, 621-645 MR2025276 (2004m:34204)

[Sz] G. Szego, Orthogonal polynomials, AMS, Providence, Rhode Island, 3d edition, 1967 MR0310533 (46:9631)

[V1] A. Varchenko, Theorems of Topological Equisingularity of Families of Algebraic Manifold and Polynomial Mappings, Izv. Acad. Sci. USSR, 36 (1972), 957-1019 MR0337956 (49:2725)

[V2] A. Varchenko, Critical points of the product of powers of linear functions and families of bases of singular vectors, Compos. Math., 97 (1995), 385-401 MR.1353281 (96j:32053)

Department of Mathematics, Indiana University-Purdue University-Indianapolis, 402 N. Blackford St., LD 270, Indianapolis, Indiana 46202

E-mail address: mukhin@math.iupui.edu

Department of Mathematics, University of North Carolina at Chapel Hill, Chapel Hill, North Carolina 27599-3250

E-mail address: anv@email.unc.edu 